\title{
Distribution function approach to redshift space distortions. Part II:N-body simulations
}

\author{
Okumura, Teppei ; Seljak, Uroš ; McDonald, Patrick ; Desjacques, Vincent
}

\begin{abstract}
Measurement of redshift-space distortions (RSD) offers an attractive method to directly probe the cosmic growth history of density perturbations. A distribution function approach where RSD can be written as a sum over density weighted velocity moment correlators has recently been developed. In this paper we use results of N-body simulations to investigate the individual contributions and convergence of this expansion for dark matter. If the series is expanded as a function of powers of , cosine of the angle between the Fourier mode and line of sight, then there are a finite number of terms contributing at each order. We present these terms and investigate their contribution to the total as a function of wavevector k. For 2 the correlation between density and momentum dominates on large scales. Higher order corrections, which act as a Finger-of-God (FoG) term, contribute $1 \%$ at k $0.015 \mathrm{hMpc}-1,10 \%$ at $\mathrm{k} \quad 0.05 \mathrm{hMpc}-1$ at $\mathrm{z}=0$, while for $\mathrm{k}>0.15 \mathrm{hMpc}-1$ they dominate and make the total negative. These higher order terms are dominated by density-energy density correlations which contributes negatively to the power, while the contribution from vorticity part of momentum density auto-correlation adds to the total power, but is an order of magnitude lower. For 4 term the dominant term on large scales is the scalar part of momentum density auto-correlation, while higher order terms dominate for $\mathrm{k}>$ $0.15 \mathrm{hMpc}-1$. For 6 and 8 we find it has very little power for $\mathrm{k}<0.15 \mathrm{hMpc}-1$, shooting up by $2-3$ orders of magnitude between $\mathrm{k}<0.15 \mathrm{hMpc}-1$ and $\mathrm{k}<0.4 \mathrm{hMpc}-1$. We also compare the expansion to the full 2-d Pss $(\mathrm{k}$, ), as well as to the monopole, quadrupole, and hexadecapole integrals of Pss(k, ). For these statistics an infinite number of terms contribute and we find that the expansion achieves percent level accuracy for $\mathrm{k}<0.15 \mathrm{hMpc}-1$ at 6 -th order, but breaks down on smaller scales because the series is no longer perturbative. We explore resummation of the terms into FoG kernels, which extend the convergence up to a factor of 2 in scale. We find that the FoG kernels are approximately Lorentzian with velocity dispersions around $600 \mathrm{~km} / \mathrm{s}$ at $\mathrm{z}=0$.
\end{abstract}

DOI: https://doi.org/10.1088/1475-7516/2012/02/010

Posted at the Zurich Open Repository and Archive, University of Zurich

ZORA URL: https://doi.org/10.5167/uzh-70087

Journal Article

Originally published at:

Okumura, Teppei; Seljak, Uroš; McDonald, Patrick; Desjacques, Vincent (2012). Distribution function approach to redshift space distortions. Part II:N-body simulations. Journal of Cosmology and Astroparticle Physics, 2012(2):10.

DOI: https://doi.org/10.1088/1475-7516/2012/02/010 


\title{
Distribution function approach to redshift space distortions. Part II: $N$-body simulations
}

\section{Teppei Okumura, ${ }^{a}$ Uroš Seljak, ${ }^{a, b, c, d}$ Patrick McDonald, ${ }^{b, e}$ and Vincent Desjacques ${ }^{d}$}

${ }^{a}$ Institute for the Early Universe, Ewha Womans University, Seoul 120-750, S. Korea

${ }^{b}$ Department of Physics and Lawrence Berkeley National Laboratory, University of California, Berkeley, California 94720, USA

${ }^{c}$ Department of Astronomy, University of California, Berkeley, California 94720, USA

${ }^{d}$ Institute of Theoretical Physics, University of Zurich, 8057 Zurich, Switzerland

${ }^{e}$ Physics Dept., Brookhaven National Laboratory, Building 510A, Upton, NY 11973-5000, USA

E-mail: teppei@ewha.ac.kr, useljak@berkeley.edu,pvmcdonald@lbl.gov,

dvince@physik.uzh.ch

\begin{abstract}
Measurement of redshift-space distortions (RSD) offers an attractive method to directly probe the cosmic growth history of density perturbations. A distribution function approach where RSD can be written as a sum over density weighted velocity moment correlators has recently been developed. In this paper we use results of N-body simulations to investigate the individual contributions and convergence of this expansion for dark matter. If the series is expanded as a function of powers of $\mu$, cosine of the angle between the Fourier mode and line of sight, then there are a finite number of terms contributing at each order. We present these terms and investigate their contribution to the total as a function of wavevector $k$. For $\mu^{2}$ the correlation between density and momentum dominates on large scales. Higher order corrections, which act as a Finger-of-God (FoG) term, contribute $1 \%$ at $k \sim 0.015 h \mathrm{Mpc}^{-1}, 10 \%$ at $k \sim 0.05 h \mathrm{Mpc}^{-1}$ at $z=0$, while for $k>0.15 h \mathrm{Mpc}^{-1}$ they dominate and make the total negative. These higher order terms are dominated by density-energy density correlations which contributes negatively to the power, while the contribution from vorticity part of momentum density auto-correlation adds to the total power, but is an order of magnitude lower. For $\mu^{4}$ term the dominant term on large scales is the scalar part of momentum density auto-correlation, while higher order terms dominate for $k>0.15 h \mathrm{Mpc}^{-1}$. For $\mu^{6}$ and $\mu^{8}$ we find it has very little power for $k<0.15 h \mathrm{Mpc}^{-1}$, shooting up by 2-3 orders of magnitude between $k<0.15 h \mathrm{Mpc}^{-1}$ and $k<0.4 h \mathrm{Mpc}^{-1}$. We also compare the expansion to the full 2 -d $P^{s s}(k, \mu)$, as well as to the monopole, quadrupole, and hexadecapole integrals of $P^{s s}(k, \mu)$. For these statistics an infinite number of terms contribute and we find that the expansion achieves percent level accuracy for $k \mu<0.15 h \mathrm{Mpc}^{-1}$ at 6 -th order, but breaks down on smaller scales because the series is no longer perturbative. We explore resummation of the terms into FoG kernels, which extend the convergence up to a factor of 2 in scale. We find that the FoG kernels are approximately Lorentzian with velocity dispersions around $600 \mathrm{~km} / \mathrm{s}$ at $z=0$.
\end{abstract}

Keywords: galaxy clustering, power spectrum, redshift surveys

ArXiv ePrint: 1109.1609 


\section{Contents}

1 Introduction $\quad 1$

2 Redshift-space distortions from the distribution function $\quad 2$

2.1 Angular dependence 3

3 Numerical analysis $\quad 4$

$3.1 \quad N$-body simulations $\quad 4$

3.2 Matter power spectrum $\quad 4$

$\begin{array}{ll}3.3 & \text { Legendre expansion } \\ \end{array}$

4 Fingers-of-God resummation $r$

4.1 Testing the Fingers-of-God model $r$

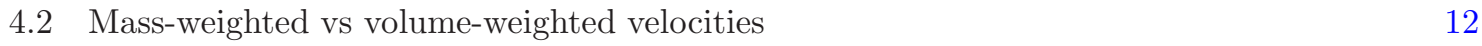

5 Expansion in powers of $\mu^{2} \quad 13$

$\begin{array}{ll}5.1 \mu^{2} \text { terms } & 14\end{array}$

$\begin{array}{ll}5.2 \mu^{4} \text { terms } & 15\end{array}$

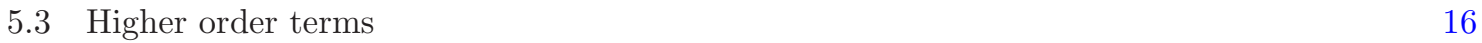

6 Conclusions $r$

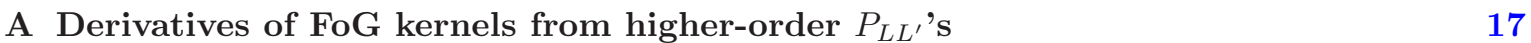

\section{Introduction}

Galaxy redshift surveys are one of the most powerful tools to probe cosmological models [1]. One way to extract the information is from the shape of the power spectrum or correlation function, assuming it traces the underlying dark matter. Another method involves baryonic acoustic oscillations (BAOs), detected in various redshift surveys, which enables one to measure angular diameter distance and compare it to the same quantity measured in cosmic microwave background. This in turn probes the expansion history of the Universe and allows to study the nature of dark energy [e.g., 2, 3]. Third piece of information in redshift surveys comes from redshift space distortions (RSD): the observed galaxy distribution is distorted along the line of sight due to the Doppler shifts caused by peculiar velocities [4-6]. In linear theory this allows one to measure the rate of growth of structure, which allows for another way to measure the matter content of the universe, including the amount and nature of dark energy. The last two methods are complementary: cosmological models in different gravity theories with the same expansion history cannot be distinguished by the distance scales of BAOs, but can if growth of structure is also measured [e.g., 7-9]. Additional information is obtained by Alcock-Paczyński test [10-12].

RSD have been analyzed in many galaxy surveys to determine the cosmological models [e.g., 13-22]. However, it was shown by [23-26] that the growth rate reconstructed from the redshift-space distortions can have scale dependent biases, which indicate a breakdown of linear theory predictions. These effects show up on relatively large scales, suggesting one must go beyond the linear theory in the analysis of RSD. This will become even more important in the future, with several ongoing and upcoming galaxy surveys that will measure RSD to a high precision [27-31].

Given the high precision of the future surveys, correspondingly more accurate theoretical predictions become essential for their interpretation. As was emphasized by [32], there are important nonlinear effects that need to be addressed in order to achieve accurate theoretical predictions. In order to account for the nonlinearity of the gravitational evolution, standard perturbation theory has long been used to describe the power spectrum at quasi-nonlinear scales [e.g., 33]. Recently there have 
been many studies to predict the power spectrum in nonlinear regime beyond the framework of the standard perturbation theory (SPT) [34-39]. These different approaches were compared to the $N$ body simulations in [40,41]. Similarly, initial RSD work was based on the lowest order SPT [42-45]. However, as pointed out by $[32,43]$, SPT in redshift space breaks down at larger scales than in real space because of nonlinear redshift distortion effects, sometimes called Finger-of-God (FoG) effect [4]. Recent development using more sophisticated perturbation methods applicable to the redshift-space power spectrum includes [46-48]. [22] present detailed comparisons of these predictions with the observed galaxy data.

Recent paper [49] has shown that one can write the Fourier mode of density in redshift space as a sum over mass weighted moments of radial velocity, which are integrals of powers of velocity over the momentum part of the phase space distribution function. The corresponding RSD power spectrum can be written as a sum over auto and cross-correlators of these moments. This series always converges for sufficiently small expansion parameter defined below. We will use the Fourier description in this paper and scale is expressed in terms of wavevector amplitude $k$, while angular dependence is expressed in terms of $\mu$, cosine of the angle between the line of sight and Fourier mode. The expansion parameter depends on the product of the two $k_{\|}=k \mu$. It has been shown in [49] that the moments can be decomposed into helicity eigenstates, which are eigenmodes under rotation around direction of $\mathbf{k}$ vector. Only equal helicity eigenstates correlate, leading to a specific angular structure of the correlators. This analysis shows that if one expands the series into powers of $\mu^{2}$, a finite number of terms contribute at each (finite) order. This suggests that RSD can be better understood in terms of this expansion rather than the Legendre moments usually used [49]. On the other hand, Legendre moments are uncorrelated in real observations, while powers of $\mu^{2}$ are not, leading to correlations between the higher and lower orders. We will pursue both approaches here.

This is the second paper in a series studying the redshift-space distortions based on a distribution function approach, following the theory and angular decomposition presented in [49]. In this paper we test the formalism to describe the redshift-space power spectrum in nonlinear regime using a large set of cosmological $N$-body simulations, as well as present the individual terms of expansion for comparison against each other. We focus on the dark matter in this paper, leaving the application to halos and galaxies to future work. The structure of this paper is as follows. In section 2 we briefly describe the distribution function approach to RSD. Then we apply it to simulations to test this expansion and show the contributions from individual terms: in section 3 we first show the contributions to the 2 -d power spectrum in redshift space, then proceed to Legendre moments. We discuss the FoG modeling in section 4 and present an attempt to compare the expansion to one in terms of volume weighted quantities. Finally in section 5 we apply the method to powers of $\mu^{2}$ expansion, which we argue is a more natural way to expand 2-d information, showing individual contributions to the lowest order terms. This is followed by conclusions in section 6 .

\section{Redshift-space distortions from the distribution function}

The exact evolution of collisionless particles is described by the Vlasov equation [1]. Following the discussion by [50], we start from the distribution function of particles $f(\mathbf{x}, \mathbf{q}, t)$ at phase-space position $(\mathbf{x}, \mathbf{q})$ in order to derive the perturbative redshift-space distortions. Here $\mathbf{x}$ is the comoving position and $\mathbf{q}=\mathbf{p} / a$ is the comoving momentum ( $\mathbf{p}$ is the proper momentum and $a$ is the scale factor). The density field in redshift space is related to moments of distribution function as

$$
\delta_{s}(\mathbf{k})=\sum_{L=0} \frac{1}{L !}\left(\frac{i k_{\|}}{H}\right)^{L} T_{\|}^{L}(\mathbf{k})
$$

where $H$ is the Hubble parameter and $T_{\|}^{L}(\mathbf{k})$ is the Fourier transform of $T_{\|}^{L}(\mathbf{x})$, defined as

$$
T_{\|}^{L}(\mathbf{x})=\frac{m}{\bar{\rho}} \int d^{3} \mathbf{q} f(\mathbf{x}, \mathbf{q}) u_{\|}^{L}=\left\langle(1+\delta(\mathbf{x})) u_{\|}^{L}(\mathbf{x})\right\rangle_{\mathbf{x}},
$$

where $u_{\|}$is the radial comoving velocity, $m u_{\|}=q_{\|}=\mathbf{q} \cdot \hat{r}, m$ is the particle mass, $\hat{r}$ is the unit vector pointing along the observer's line of sight and $\bar{\rho}$ is the mean mass density. 
The power spectrum in redshift space is given by [49]

$$
P^{s s}(\mathbf{k})=\sum_{L=0}^{\infty} \frac{1}{L !^{2}}\left(\frac{k \mu}{H}\right)^{2 L} P_{L L}(\mathbf{k})+2 \sum_{L=0}^{\infty} \sum_{L^{\prime}>L} \frac{(-1)^{L}}{L ! L^{\prime} !}\left(\frac{i k \mu}{H}\right)^{L+L^{\prime}} P_{L L^{\prime}}(\mathbf{k})
$$

where $k_{\|} / k=\cos \theta=\mu$. It is useful to compare this to Kaiser's linear theory prediction [5, 32]. Thus we have

$$
P_{\text {Kaiser }}^{s s}(\mathbf{k})= \begin{cases}\left(1+f \mu^{2}\right)^{2} P_{\text {lin }}(k) & ; \text { linear } \\ P_{00}+2 f \mu^{2}\left(\frac{i k}{H \mu f}\right) P_{01}+f^{2} \mu^{4}\left(\frac{k}{H \mu f}\right)^{2} P_{11} & ; \text { nonlinear }\end{cases}
$$

where $P_{\text {lin }}$ denotes the linear power spectrum and $f=d \ln D / d \ln a$ with $D$ the growth factor. These terms will in general have nonlinear corrections, so we call this approximation the nonlinear Kaiser order approximation. Replacing these lowest 3 moments with the standard linear theory we obtain the original linear Kaiser model of equation (2.4). Here we want to view this series simply as a series in $k_{\|}$, investigating the convergence as more terms are added.

Note that the calculations never require anything but simple power spectra of mass-weighted powers of velocity to be computed from the simulations. As we will compare RSD power spectrum to the sum from individual terms there should not be much sampling variance in the comparison, because both are calculated from the same simulation, so the large-scale fluctuations will be the same. The order of $k_{\|}=k \mu$ needed for convergence to a given level of accuracy will inevitably increase as one goes to increasingly small scales, with the whole expansion eventually breaking down once $k \mu \sigma / H>1$, where $\sigma$ is a typical (comoving) velocity of the system. We will see in section 3 that the nonlinear terms of $P_{L L^{\prime}}$, particularly $P_{02}$, have significant contributions even at the scales larger than the breakdown scale.

\subsection{Angular dependence}

By performing helicity decomposition [49] show that the power spectrum can be written as

$$
P_{L L^{\prime}}(\mathbf{k})=\sum_{(l=L, L-2, . .)} \sum_{\left(l^{\prime}=L^{\prime}, L^{\prime}-2, . . ;\right.} \sum_{\left.l^{\prime} \geq l\right)}^{l} P_{m=0}^{L, L^{\prime}, m}(k) P_{l}^{m}(\mu) P_{l^{\prime}}^{m}(\mu)
$$

where $P_{l}^{m}(\mu)$ are the associated Legendre polynomials, which determine the angular dependence of the spherical harmonics. There are 5 numbers that describe these objects: $L$ and $L^{\prime}$ describe the power of two velocity moments we are correlating, $l, l^{\prime}$ describe the rank of the object, for example $l=1$ is rank-1, which is a 3 -d vector, $l=2$ is a 3 -d tensor etc. Finally, $m$ is the helicity eigennumber, which ranges between 0 and $l\left(l \leq l^{\prime}\right)$. Only equal helicity components of expansion have a non-vanishing correlator. There is a close relation between the order of the moments and their angular dependence. The lowest contribution in powers of $\mu$ to $P^{s s}(k)$ is $\mu^{L+L^{\prime}}$ if $L+L^{\prime}$ is even or $\mu^{L+L^{\prime}+1}$ if $L+L^{\prime}$ is odd, and the highest is $\mu^{2\left(L+L^{\prime}\right)}$. Thus for $P_{00}(\mathbf{k})$ the only angular term is isotropic term $\left(\mu^{0}\right)$, for $P_{01}(\mathbf{k})$ the only angular term is $\mu^{2}, P_{11}(\mathbf{k})$ and $P_{02}(\mathbf{k})$ contain both $\mu^{2}$ and $\mu^{4}$ etc. Note that only even powers of $\mu$ enter in the final expression, as required by the symmetry. We can thus write

$P^{s s}(\mathbf{k})=\sum_{L=0}^{\infty} \frac{1}{L !^{2}}\left(\frac{k}{H}\right)^{2 L} \sum_{j=2 L}^{4 L} P_{L L}^{(j)}(\mathbf{k}) \mu^{j}+2 \sum_{L=0}^{\infty} \sum_{L^{\prime}>L} \frac{(-1)^{L}}{L ! L^{\prime} !}\left(\frac{i k}{H}\right)^{L+L^{\prime}} \sum_{j=\left(L+L^{\prime}\right) \operatorname{or}\left(L+L^{\prime}+1\right)}^{2\left(L+L^{\prime}\right)} P_{L L^{\prime}}^{(j)}(\mathbf{k}) \mu^{j}$,

so that terms $P_{L L^{\prime}}^{(j)}$ are coefficients in expansion in powers of $\mu^{j}$ of contributions of $L, L^{\prime}$ terms to $P^{s s}$. Since the $j$ index has to be even, the lowest order is either $L+L^{\prime}$ or $L+L^{\prime}+1$, whichever is even, thus the higher order terms also have higher order powers of $\mu^{j}$ and one can in principle separate them from lower order terms. These terms can be uniquely extracted from simulations from angular dependence of $P_{L L^{\prime}}$ terms and so we will focus on them, although sometimes it is useful to decompose them into the individual helicity eigenstates instead. 


\section{$3 \quad$ Numerical analysis}

\subsection{N-body simulations}

The power spectra of the derivative expansion are all from mass-weighted velocity moments and thus can be straightforwardly measured from simulations. We use a series of $N$-body simulations of the $\Lambda$ CDM cosmology seeded with Gaussian initial conditions, which is an updated version of [51]. The primordial density field is generated using the matter transfer function by CMBFAST [52]. We adopt the standard $\Lambda$ CDM model with the mass density parameter $\Omega_{m}=0.279$, the baryon density parameter $\Omega_{b}=0.0462$, the Hubble constant $h=0.7$, the spectral index $n_{s}=0.96$, and a normalization of the curvature perturvations $\Delta_{\mathcal{R}}^{2}=2.21 \times 10^{-9}$ (at $k=0.02 \mathrm{Mpc}^{-1}$ ) which gives the density fluctuation amplitude $\sigma_{8} \approx 0.81$, which are the best-fit parameters in the WMAP 5-year data [53]. We employ $1024^{3}$ particles of mass $3.0 \times 10^{11} h^{-1} M_{\odot}$ in a cubic box of side $1600 h^{-1} \mathrm{Mpc}$. The positions and velocities of all the dark matter particles are output at $z=0,0.509,0.989$, and 2.070, which are quoted as $z=0,0.5,1$, and 2 in what follows for simplicity. We use 12 independent realizations in order to reduce the statistical scatters. For the detail of the simulations see [51].

Next we describe how we measure the power spectra from our simulation samples. We assign the density field and the mass-weighted velocity moments in real space on $1024^{3}$ grids using a cloudin-cell interpolation method according to the positions of particles. To directly measure $P^{s s}(\mathbf{k})$ we also need the density field in redshift space. In measuring the redshift-space density field, we distort the positions of particles along the line-of-sight according to their peculiar velocities before we assign them to the grid. We regard each direction along the three axes of simulation boxes as the line of sight and the statistics are averaged over three projections of all realizations for a total of 36 samples. We use a fast Fourier transform to measure the Fourier modes of the density fields in real space $\delta(\mathbf{k})$ and in redshift space $\delta_{s}(\mathbf{k})$, as well as the mass-weighted velocity moment fields in real space $T_{\|}^{L}(\mathbf{k})$. Finally, the power spectrum in redshift space, $P^{s s}(\mathbf{k})$, as well as the power spectra of the mass-weighted velocity moments $P_{L L^{\prime}}(\mathbf{k})$, are measured by multiplying the modes of the two fields (or squaring in case of auto-correlation) and averaging over the Fourier modes. Throughout this paper, we neglect shot noise because we have sufficient number of dark matter particles and such an effect is thus negligibly small. Error bars in the following results are estimated from bootstrap resampling. The dispersion in power spectra measurements is large on large scales because of sampling variance, but it is mostly eliminated by taking the ratio of the two spectra obtained from the same set of realizations (e.g. [54]).

\subsection{Matter power spectrum}

In this subsection we first measure the redshift-space power spectrum, $P^{s s}(k, \mu)$, directly in redshift space, which we call the "reference" power spectrum. The reference power spectrum in redshift space is shown as functions of $(k, \mu)$ at $z=0$ and 2 as the red lines in figure 1 . We adopt the constant $\mu$ binning into five bins between $0 \leq \mu \leq 1$, but only three $\mu$ bins among the five are plotted. In figure 1 we also show contributions of the terms of $P_{L L^{\prime}}$ for $\left(0 \leq L+L^{\prime} \leq 4\right)$ to $P^{s s}(k, \mu)$ computed from the mass-weighted velocity moments. At $\mu \sim 0$ contributions from the higher order power spectra of the velocity moments are small and $P^{s s} \simeq P_{00}$ because each $P_{L L^{\prime}}$ is multiplied by a factor of $(k \mu)^{L+L^{\prime}}$.

On large scales one expects $P_{00}$ to be followed by the other two linear order terms, which are $P_{01}$ and the scalar part of $P_{11}$, i.e. $P_{11}^{110}$. Note however that the latter scales as $\mu^{4}$, while there are two nonlinear terms, the vector part of $P_{11}, P_{11}^{111}$, and the scalar part of $P_{02}$, which itself has two terms, both helicity 0 , one from energy density correlated with the density $P_{00}^{020}$, and one from anisotropic stress density correlated with density, $P_{02}^{020}$, that contain terms which scale as $\mu^{2}$. As a result, for sufficiently low $\mu$ these nonlinear terms dominate over the linear term in $P_{11}^{110}$ even on very large scales. For example, for $\mu=0.1$ at $z=0$ we see that $P_{02}$ dominates over $P_{11}$ on all scales probed, despite the fact that $P_{11}$ contains a linear order term. We also see that $P_{11}$ does not follow the linear theory on all but the largest scales, but instead has the shape dependence similar to $P_{02}$, characteristic of the nonlinear terms. As pointed out in [49], the nonlinear helicity 1 (vector or vorticity) terms in $P_{11}$ are closely connected to $P_{02}$ and partially cancel each other. This angular decomposition is discussed further below in section 5 , where we present the individual helicity terms separately. 


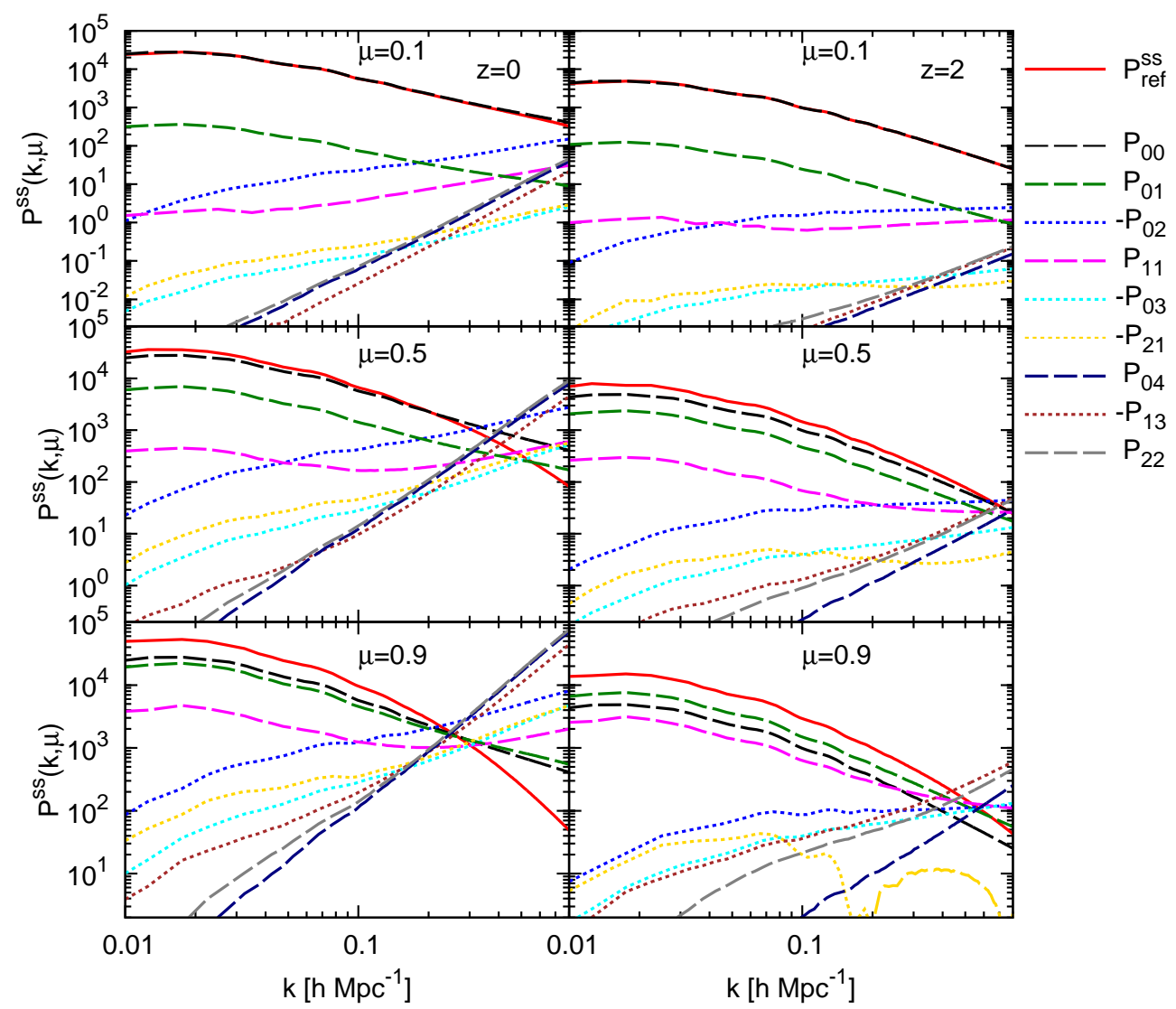

Figure 1. Power spectra measured in redshift space $P^{s s}(k, \mu)$ and individual contributions to it from the terms of the moments expansion up to 4 -th order at $z=0$ (left) and $z=2$ (right). The width of $\mu$ bin is 0.2 centered around the values shown in each panel. The solid and dashed lines show the positive values, while the dotted lines the negative values.

Because of $(k \mu)^{L+L^{\prime}}$ weight the higher-order terms scale more rapidly with $k$, and dominate on small scales: this is the region where RSD are dominated by FoG effects. One needs to take into account more and more higher-order terms in order to make the expansion (equation (2.3)) valid at such smaller scales. This effect is more significant at $z=0$ due to higher velocities. One can see that the higher order terms cross the lower order terms at $k \mu \sim 0.2 h \mathrm{Mpc}^{-1}(z=0)$ and $k \mu \sim 0.4 h \mathrm{Mpc}^{-1}(z=2)$. This is where the perturbative parameter $k \mu \sigma / H$ becomes of order unity and the perturbative approach breaks down, consistent with [32]. At that point higher order terms dominate over the lower order terms and we no longer have a convergence. This can be seen in figure 1: while for high $k$ the redshift space power spectrum $P^{s s}(k, \mu)$ decreases in power relative to the real space case $P_{00}(k)$, the individual terms in the series expansion increase due to their $(k \mu)^{L+L^{\prime}}$. This suggests that a non-perturbative approach is needed in this regime: we will explore the so-called FoG resummation in section 4 . Figure 1 suggests that the typical velocity $\sigma$ in the expansion is about $500 \mathrm{~km} / \mathrm{s}$ at $z=0$ and $250 \mathrm{~km} / \mathrm{s}$ at $z=2$. We confirm these numbers in a more detailed FoG analysis below.

\subsection{Legendre expansion}

We can compare the agreement between moments expansion and the full $P^{s s}$ as a function of the order in the series $\left(L, L^{\prime}\right)$. It is customary to expand the redshift-space power spectrum in terms of Legendre multipole moments [e.g., 22, 24, 55-57]. The motivation for this expansion is that if one uses full angular information the Legendre moments are uncorrelated. Using Legendre polynomials 
$\mathcal{P}_{l}(\mu)$, we have [58]

$$
P^{s s}(k, \mu)=\sum_{l=0,2,4, \cdots} P_{l}^{s s}(k) \mathcal{P}_{l}(\mu) .
$$

The multipole moments, $P_{l}^{s s}$, are obtained by inversion of this relation,

$$
P_{l}^{s s}(k)=\frac{2 l+1}{2} \int_{-1}^{+1} P^{s s}(k, \mu) \mathcal{P}_{l}(\mu) d \mu .
$$

Most of the studies on modeling redshift-space distortions focus on the monopole $(l=0)$ and quadrupole $(l=2)$, although hexadecapole $(l=4)$ also contains some cosmological information [57].

In figure 2 we show the monopole power spectrum at $z=0,0.5,1$, and 2 summed up to nonlinear Kaiser, 2nd, 3rd, 4th, and 6th order approximations in $k \mu$ expansion. Here we denote the summation at a given order as including all terms that have the same $L+L^{\prime}$ : hence 2nd order includes all 3 Kaiser terms $P_{00}, P_{01}$ and $P_{11}$, as well as $P_{02}$, while the nonlinear Kaiser model includes only the first three. The lower panels show the error for a given order, $P_{0, \text { sum }}^{s s} / P_{0, \text { ref }}^{s s}-1$. The linear theory power spectra with the input cosmological parameters of our simulations are also plotted for comparison. The power spectrum of the nonlinear Kaiser model starts to deviate from the reference spectrum at very large scales, $k \simeq 0.05 h \mathrm{Mpc}^{-1}$. However, adding the term $P_{02}$, which has the same order contribution as $P_{11}$, to the nonlinear Kaiser model, improves the accuracy. Adding the higher order terms continues to improve the accuracy down to smaller and smaller scales, but the gains decrease as we approach the scale $k=\sigma^{-1} \sim 0.2 h \mathrm{Mpc}^{-1}(z=0)$, where the perturbative expansion breaks down. Our formula for the redshift-space monopole spectrum $P_{0 \text {,sum }}^{s s}$, summed up to or more than 6th order, is accurate within a few percent accuracy at $k \simeq 0.2 h \mathrm{Mpc}^{-1}$ at $z=0$ and at $k \simeq 0.4 \mathrm{~h} \mathrm{Mpc}^{-1}$ at $z=2$. It predicts not only the overall shape of the redshift-space power spectrum up to these scales but also baryon acoustic oscillations (BAO): to see this more clearly we show the summed power spectra divided by the smoothed no-wiggle spectrum [59] in figure 3 .

Figure 4 is the same as figure 2, but shows the results for the quadrupole spectra $P_{2}^{s s}$. Because the nonlinear quadrupole spectra crosses zero at high- $k$, there exists a singularity point for the ratio of summed and reference spectra at small scales. The predictions for the quadrupole moment reproduce the reference spectrum within a few percents up to the scales of the singular point, $k \simeq 0.15 h \mathrm{Mpc}^{-1}$ at $z=0$ and $k \simeq 0.3 h \mathrm{Mpc}^{-1}$ at $z=2$. The quadrupole spectra divided by the corresponding nowiggle spectrum are shown at the right side of Figure 3 . Figure 5 shows the results for the hexadecapole spectrum. We adopt broader $k$ binning for the hexadecapole moment at $k<0.1 h \mathrm{Mpc}^{-1}$ and put artificial cuts for the plots of $k P_{4}^{s s}$ because of large sampling variance. We do not show the results obtained from the nonlinear Kaiser, 2nd and 3rd order approximations in lower panels because they strongly deviate from the reference power spectrum (as shown in the upper panels of figure 5). Although the measurement of the hexadecapole moment of the redshift-space power spectrum is very noisy, the higher order expansion predictions give a good agreement if we consider summation to 6th order.

\section{Fingers-of-God resummation}

It is clear from the results in previous section that while for $k \mu \sigma / H<1$ we have a convergence and only a finite number of terms need to be considered, there is no convergence for $k \mu \sigma / H>1$ : individual terms become larger and larger as we go to higher orders, yet the total sum in $P^{s s}(k, \mu)$ remains well behaved. This suggests we need to explore ways to resum the terms.

While it is difficult to make progress in general terms, there are specific situations that can be controlled. We are interested in a situation where pieces of terms disconnect within the correlation function. For example, in $P_{02}$ term we correlate $\delta$ with $(1+\delta) u_{\|}^{2}$, and if $u_{\|}^{2}$ is dominated by the small scales, as will be the case in systems with large velocity dispersion caused by nonlinear gravitational collapse, then on large scales this term becomes $P_{02} \sim P_{00} \sigma^{2}$, where $\sigma^{2}=\left\langle u_{\|}^{2}\right\rangle$ and the total contribution to $P^{s s}$ is $-P_{00}(k \mu \sigma / H)^{2}$. We see that this term scales as the linear order term, but has 


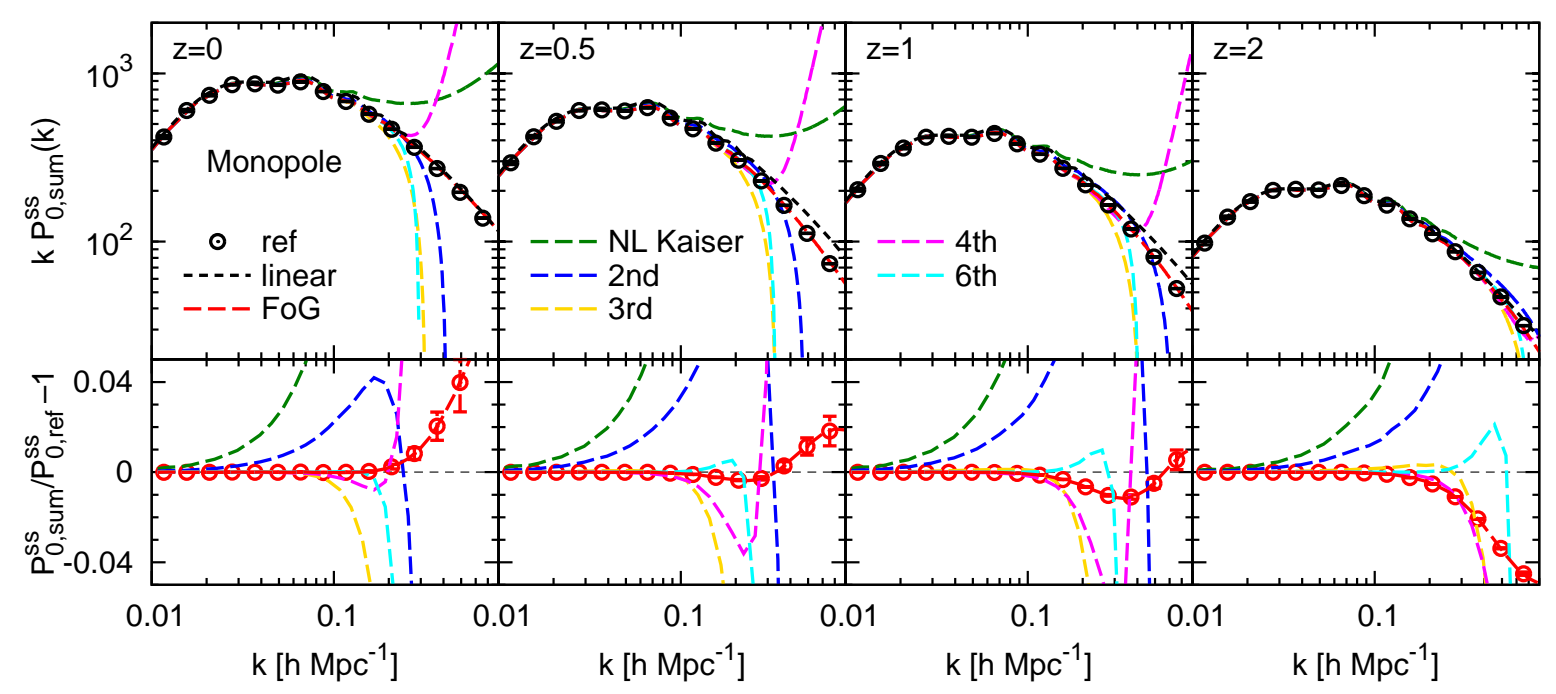

Figure 2. Upper panels: we show monopole moments of power spectrum in redshfit space $P^{s s}$. The vertical axis is multiplied by $k$ to clearly illustrate departures from a reference power spectrum. The green, blue, yellow, magenta and cyan lines respectively show our model prediction up to nonlinear Kaiser, 2nd, 3rd, 4th and 6 th order corrections, measured from the simulations. The black lines are linear theory prediction. The black points with errorbars show the reference power spectrum. The red lines show our FoG model (section 4). Lower panels: error between the summed power spectrum and the reference spectrum. The meaning of the color of each line is the same as that of the upper panels. For reference errorbars are shown for the result of our FoG model.

opposite sign to it, i.e. This term suppresses power and this suppression scales as $k^{2}$. The long range correlation is entirely in the density field. While this analysis suggests the mean square velocity field enters as the physical parameter, as discussed in [49], any bulk velocity that displaces particles as a solid body will not contribute to FoG. This cancellation shows up in part of $P_{11}$ cancelling $P_{02}$, such that only the dispersion part enters into the $\sigma^{2}$ term, while the bulk part cancels.

One can identify similar terms at higher order, for example $P_{04} \sim P_{00}\left\langle u_{\|}^{4}\right\rangle$ and one can write $\left\langle u_{\|}^{4}\right\rangle=3 \sigma^{4}+\left\langle u_{\|}^{4}\right\rangle_{c}$, where $\left\langle u_{\|}^{4}\right\rangle_{c}$ is the connected part of the curtosis. There will also be a term $P_{22} \sim P_{00} \sigma^{4}$. The total contribution to $P^{s s}$ from these terms is thus

$$
\begin{aligned}
P^{s s}(k, \mu) & =P_{00}\left[1-(k \mu \sigma / H)^{2}+(k \mu \sigma / H)^{4} / 2+2(k \mu / H)^{4}\left\langle u_{\|}^{4}\right\rangle_{c} / 4 ! \cdots\right] \\
& =P_{00} e^{-(k \sigma \mu / H)^{2}+2(k \mu / H)^{4}\left\langle u_{\|}^{4}\right\rangle_{c} / 4 ! \cdots} .
\end{aligned}
$$

The same calculation can be done at the field level. We are interested in the situation where the pieces of a term like $\delta(\mathbf{x}) u_{\|}^{2}(\mathbf{x})$ are disconnected within a correlation calculation, e.g., $\left\langle X(\mathbf{y}) \delta(\mathbf{x}) u_{\|}^{2}(\mathbf{x})\right\rangle=$ $\langle X(\mathbf{y}) \delta(\mathbf{x})\rangle\left\langle u_{\|}^{2}\right\rangle+$ other terms. We can re-sum the pieces into a FoG factor

$$
G^{1 / 2}(k \mu)=\exp \left[\sum_{L=1} \frac{1}{L !}\left(\frac{i k \mu}{H}\right)^{L}\left\langle u_{\|}^{L}\right\rangle_{c}\right]
$$

where $\left\langle u_{\|}^{L}\right\rangle_{c}$ is the connected part of $\left\langle u_{\|}^{L}\right\rangle$ and note that the odd $L$ terms are zero by symmetry. The lowest order term here is just the usual Gaussian kernel $\exp \left(-k^{2} \mu^{2} \sigma^{2} / 2 H^{2}\right)$. After this re-summation we can write the redshift-space density field as

$$
\delta_{s}(\mathbf{k})=G^{1 / 2}(k \mu)\left[\delta(\mathbf{k})+\sum_{L=1} \frac{1}{L !}\left(\frac{i k \mu}{H}\right)^{L}\left[(1+\delta(\mathbf{x}))\left[u_{\|}^{L}\right]_{c}(\mathbf{x})\right]_{\mathbf{k}}\right],
$$



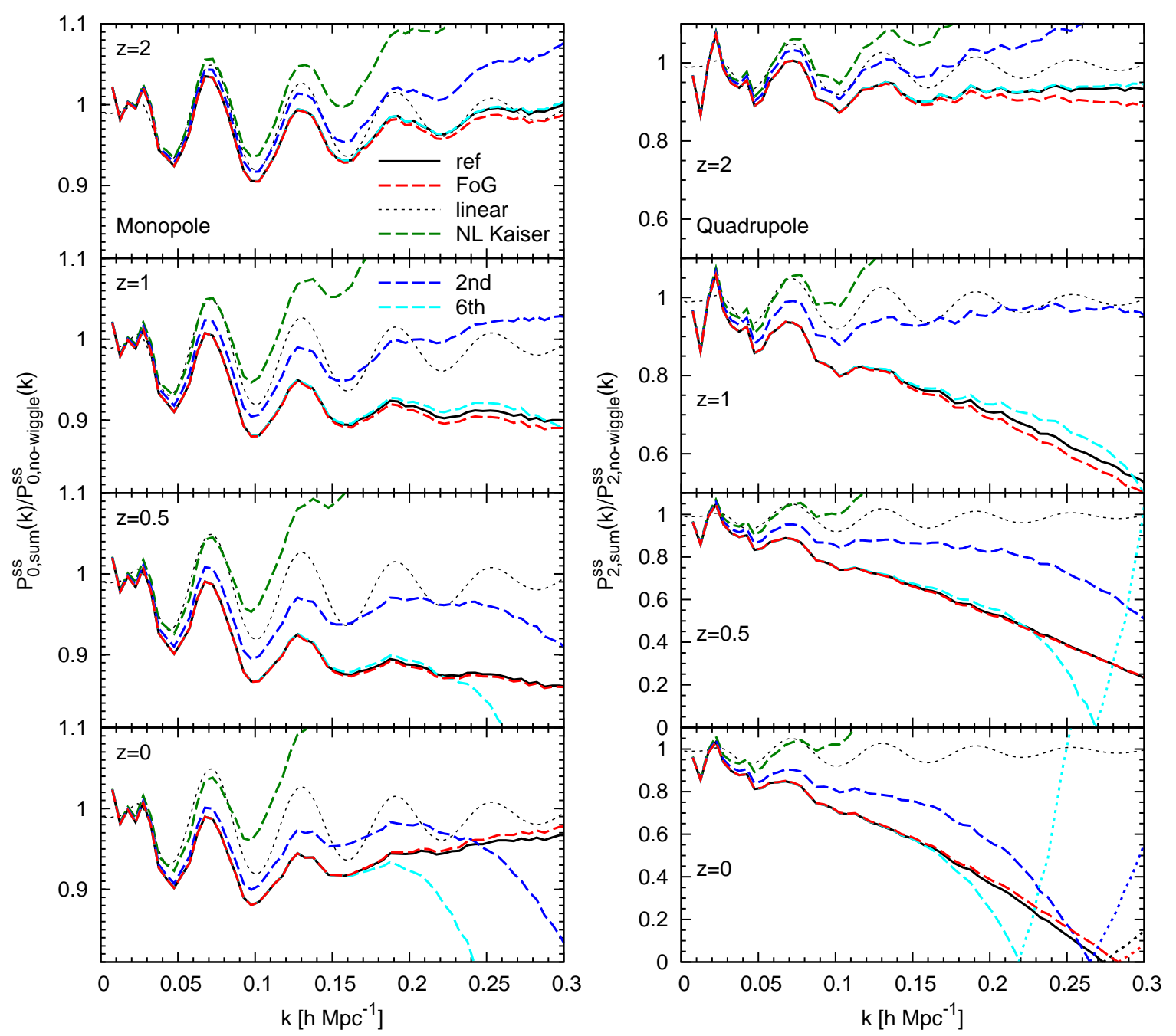

Figure 3. Redshift-space power spectrum divided by the no-wiggle approximation [59], monopole (left) and quadrupole (right). The green, blue and cyan lines show our model prediction up to nonlinear Kaiser, 2nd and 6th order corrections. The dotted black lines are linear theory prediction, while the solid black lines the reference power spectrum. The red lines show our FoG model. The negative values of the quadrupole spectrum on small scales are shown as the dotted lines.

where $\left[u_{\|}^{L}\right]_{c}$ is understood to be $u_{\|}^{L}$ minus all possible internal averages of any number of $u$ 's. This motivates us to write

$$
P^{s s}(\mathbf{k})=G\left([k \mu \sigma / H]^{2}\right) P_{\text {Kaiser }}(\mathbf{k}),
$$

where $P_{\text {Kaiser }}$ account for the lowest 3 terms given by equation (2.4) and where $G(x)$ is exponential in the simplest case where higher order reduced moments can be ignored, while more generally it is a function with alternating signs of coefficients in Taylor expansion. We have written $G(x)$ in terms of $x=[k \mu \sigma / H]^{2}$ only: if curtosis is present then we can either write it by adding additional $(k \mu)^{4}$ terms to the exponential, $\exp \left[-(k \sigma \mu / H)^{2}+2(k \mu / H)^{4}\left\langle u_{\|}^{4}\right\rangle_{c} / 4 ! \cdots\right]$, or, equivalently, we allow for a more general functional form of $G(x)$ than an exponential.

Note that in the simplest form this "derivation" gives exactly the exponential FoG form proposed 


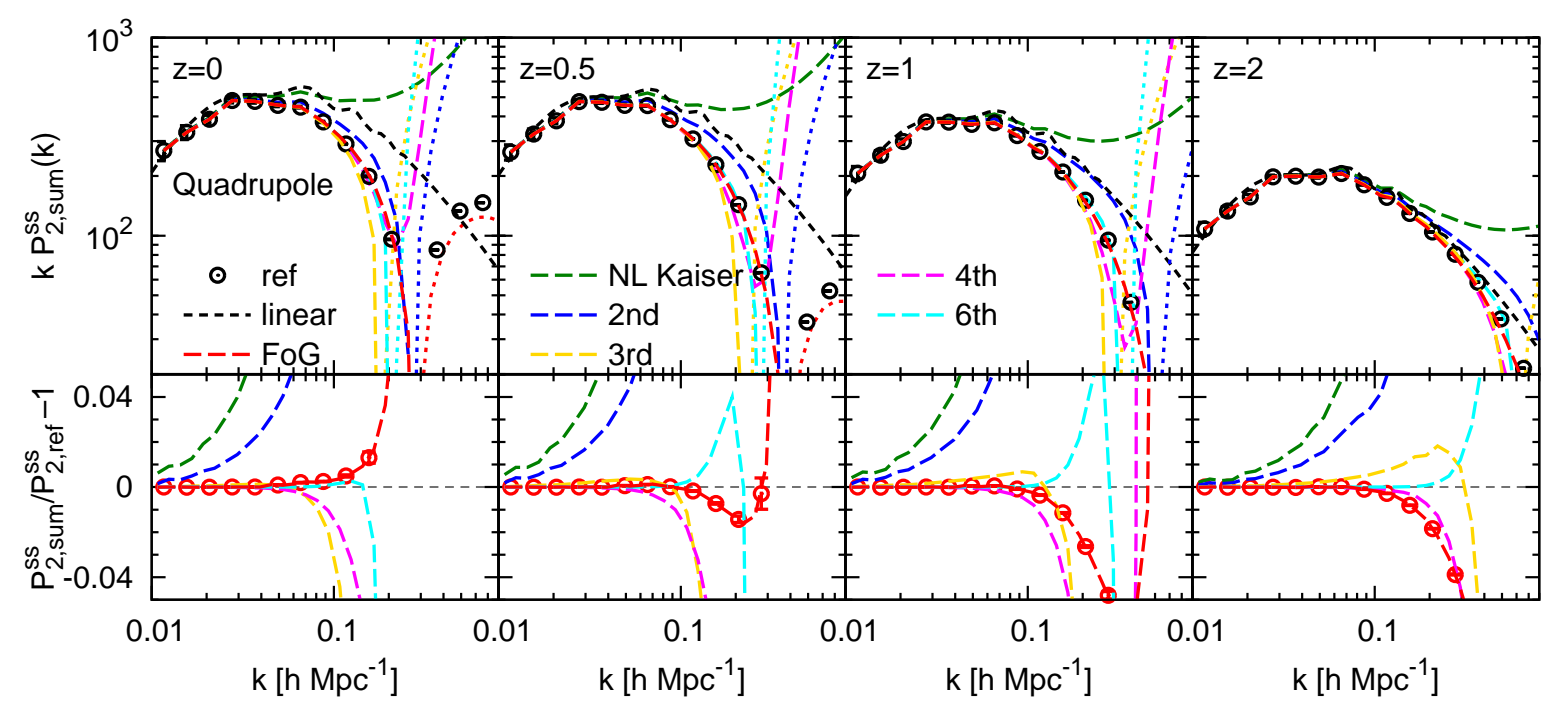

Figure 4. Same as figure 2, but for the quadrupole. The dashed lines at the top panels show positive values while the dotted lines show negative values.

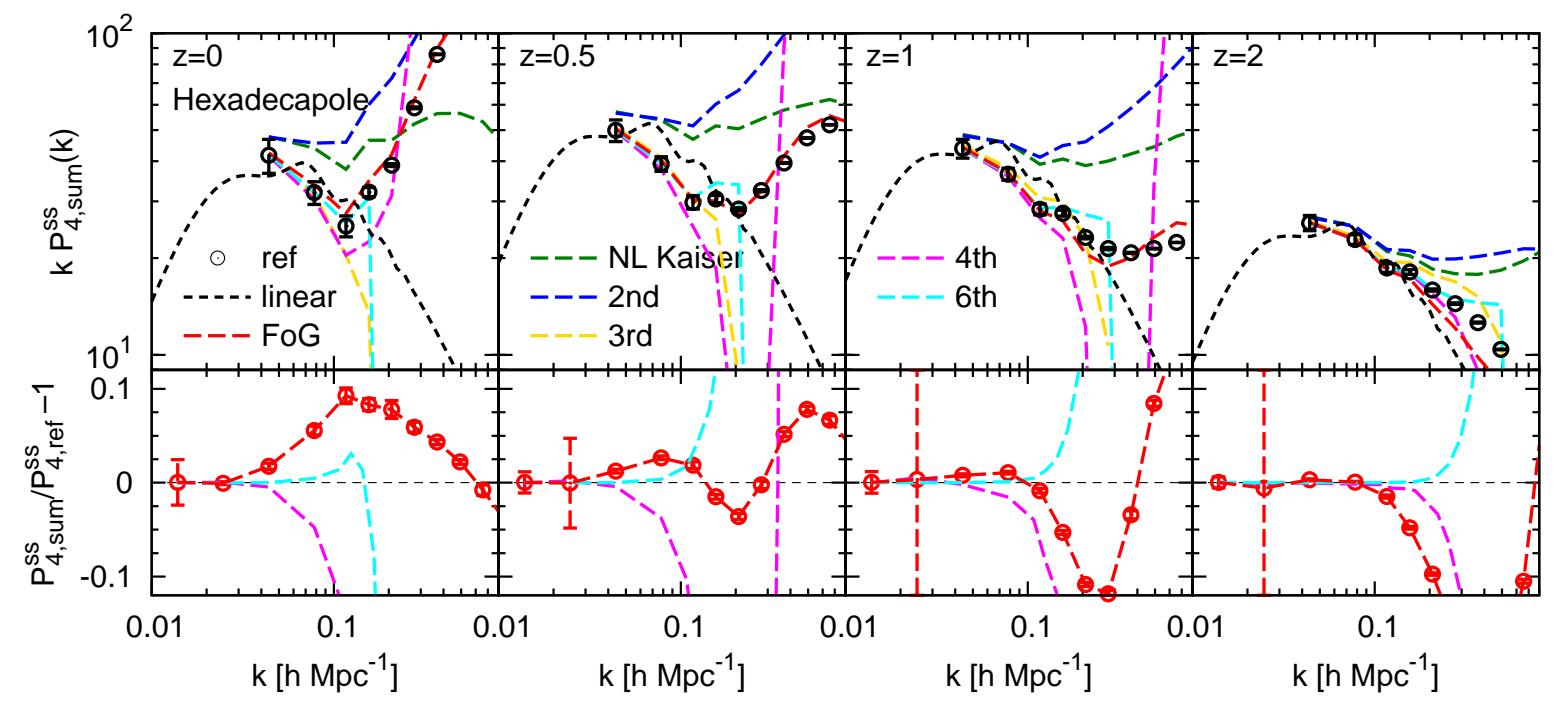

Figure 5. Same as figure 2, but for the hexadecapoles. We adopt the different bin sizes in logarithmic spacing at $k \leq 0.1 h \mathrm{Mpc}^{-1}$ and $k \geq 0.1 h \mathrm{Mpc}^{-1}$. Artificial cuts are put for the plots of the hexadecapoles at low $k$ because of large sampling variance.

in the literature $[12,32,60-62]$. Other forms for $G(x)$ have been proposed in the literature, e.g.

$$
G\left(x=(k \mu \sigma / H)^{2}\right)= \begin{cases}(1+x)^{-1} & \text { Lorentzian, } \\ \exp (-x) & \text { Gaussian }\end{cases}
$$

where $\sigma$ was treated as a free parameter. See $[26,63]$ for the studies to adopt more than one free parameter for the FoG term. These empirical forms all behave qualitively in the same manner, i.e. to first order of Taylor expansion in $(k \mu)^{2}$ they give $G=1-(k \mu \sigma / H)^{2}+\mathcal{O}\left([k \mu \sigma / H]^{4}\right)$ and higher order terms alternate in the signs.

The effective velocity dispersions can be evaluated from our expression of the redshift-space power spectrum (equation (2.3)) and uniquely resummed into the FoG kernels because the higher- 
order terms of the expansion contain all information. We know that a Taylor series of the FoG kernels like in equation (4.5) produce positive and negative terms alternatively, just like the terms in equation (2.3). However, there are a lot of $P_{L L^{\prime}}$ terms in our expansion. At each even order both positive and negative terms appear, e.g., at 4 th order the $P_{04}$ and $P_{22}$ terms have positive contributions while the $P_{13}$ term has a negative contribution. On the other hand, at odd order contributions are all positive and negative in tern, e.g., at 3rd order the terms are all negative and at 5 th order all positive. Thus we decided to include three independent FoG kernels for the two even linear order order terms $\left(P_{00}\right.$ and $\left.P_{11}\right)$ and one for the lowest odd order $P_{01}$. These three terms thus need to be individually multiplied by something which corresponds to the generalized FoG kernels. To do this, we can apply the same reasoning as the discussion around equation (4.1) to other terms that contain long range correlations between density and velocity, i.e., portions of terms such as $P_{03}$ and $P_{13}$ can be respectively written as $P_{03} \sim-6 P_{01}(k \mu \sigma / H)^{2}$ and $P_{13} \sim-3 P_{01}(k \mu \sigma / H)^{2}$, which, together with the higher order terms, can be resummed into a similar function of even orders in powers of $k \mu / H$.

Finally, we can write

$$
P^{s s}(\mathbf{k})=G_{00}\left(\left[k \mu \sigma_{00} / H\right]^{2}\right) P_{00}+2 G_{01}\left(\left[k \mu \sigma_{01} / H\right]^{2}\right) \frac{i k \mu}{H} P_{01}+G_{11}\left(\left[k \mu \sigma_{11} / H\right]^{2}\right) \frac{(k \mu)^{2}}{H^{2}} P_{11} .
$$

We have defined three different velocity dispersions $\sigma_{00}, \sigma_{01}$, and $\sigma_{11}$. The expressions of the velocity dispersions in the FoG kernels can be uniquely derived from equation (2.3) as

$$
\begin{aligned}
\sigma_{00}^{2}(\mathbf{k}) & =\frac{P_{02}}{P_{00}}, \\
\sigma_{01}^{2}(\mathbf{k}) & =\frac{1}{6} \frac{P_{03}+3 P_{21}}{P_{01}}, \\
\sigma_{11}^{2}(\mathbf{k}) & =\frac{1}{3} \frac{P_{13}}{P_{11}} .
\end{aligned}
$$

To go beyond that and determine the form of FoG kernel we expand the FoG terms as a Taylor series in terms of $(k \mu)^{2}$. Because all the phenomenological FoG models have the first derivative equal to -1 , we define $d G_{L L^{\prime}}(x) /\left.d x\right|_{x=0}=-1$. Now let us consider the ansatz for the FoG model,

$$
G_{L L^{\prime}}\left(x_{L L^{\prime}} ; \alpha_{L L^{\prime}}\right)=\left(1+\frac{x_{L L^{\prime}}}{\alpha_{L L^{\prime}}}\right)^{-\alpha_{L L^{\prime}}},
$$

where $x_{L L^{\prime}}=\left(k \mu \sigma_{L L^{\prime}} / H\right)^{2}$. Each FoG kernels contains two parameters, $\sigma_{L L^{\prime}}$ and $\alpha_{L L^{\prime}}$. These can reproduce the functional forms in previous studies: Lorentzian for $\alpha_{L L^{\prime}}=1$ and Gaussian for $\alpha_{L L^{\prime}}=\infty$. The FoG parameter $\alpha_{L L^{\prime}}$ is related to $n$-th derivative of $G_{L L^{\prime}}$ as $(n \geq 2)$

$$
\left.\frac{d^{n}}{d x^{n}} G_{L L^{\prime}}(x)\right|_{x=0}=(-1)^{n} \prod_{m=1}^{n-1}\left(1+\frac{m}{\alpha_{L L^{\prime}}}\right) .
$$

We use the $\alpha_{L L}$ 's determined from the lowest contribution at second order, as

$$
\alpha_{L L^{\prime}}(\mathbf{k})=\left[G_{L L^{\prime}}^{\prime \prime}(\mathbf{k})-1\right]^{-1}
$$

Note that the expressions for our FoG models of equation (4.6) preserve full generality up to order $(k \mu / H)^{5}$. The quality of the ansatz can be investigated by looking at higher order terms: the comparison of the derivatives of the FoG kernels up to 4 th order with the expansion terms is given in appendix A.

The FoG model in equation (4.10) is not the only option to choose. As discussed above, the resummation can be expressed more elegantly in terms of connected moments, where the cumulant theorem naturally leads to an expression of the form

$$
G_{L L^{\prime}}=\exp \left[-(k \mu \sigma / H)^{2}+(k \mu \tau / H)^{4}+\cdots\right]
$$




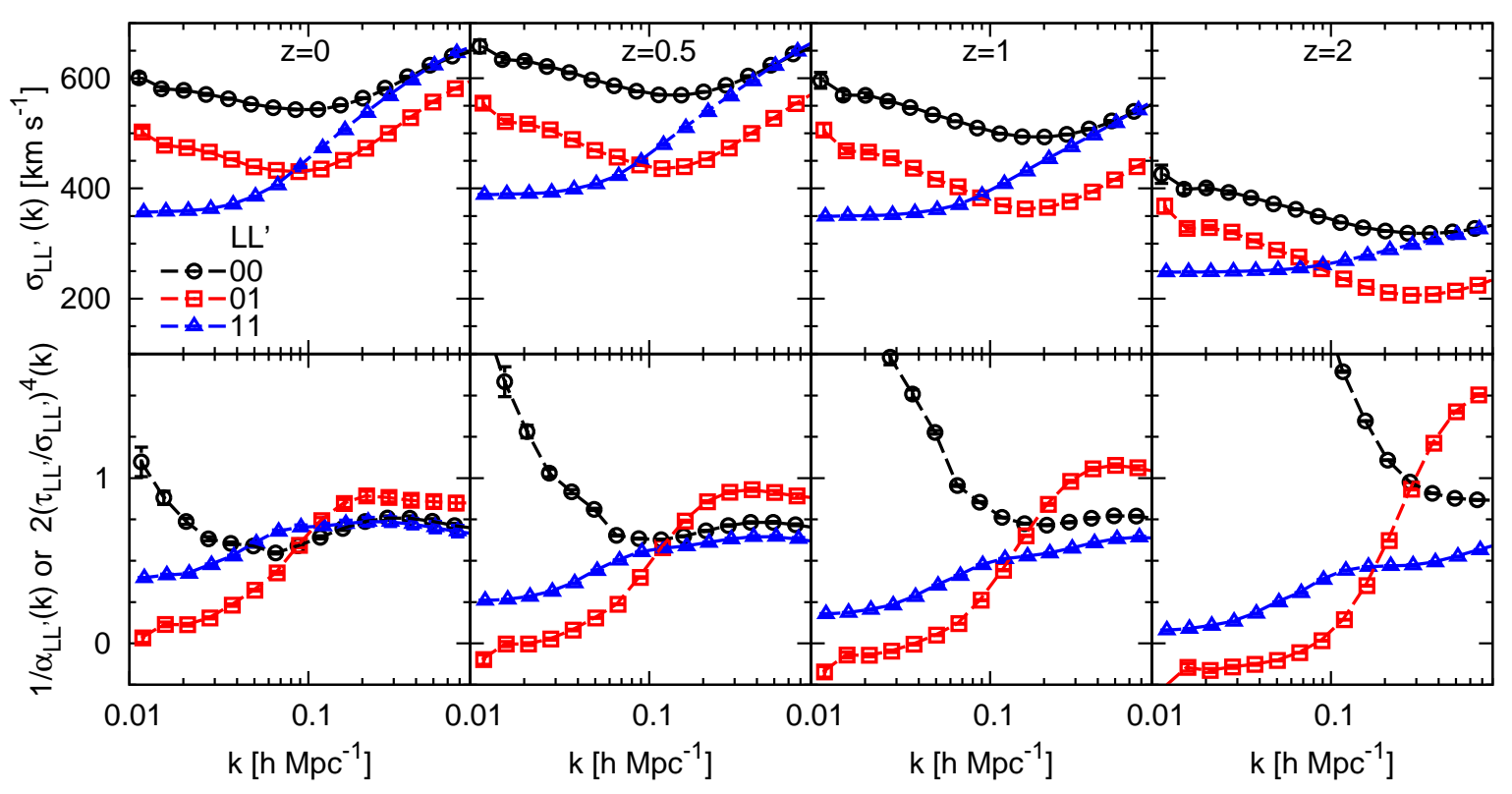

Figure 6. Spherically averaged FoG parameters, velocity dispersions (upper panels) and the power law index of FoG kernel (lower panels). The circles/triangles have been respectively offset in the negative/positive direction for clarity.

where the lowest two cumulants are variance $\sigma^{2}=\left\langle u_{\|}^{2}\right\rangle$ and kurtosis $\tau^{4}=\left\langle u_{\|}^{4}\right\rangle_{c}$, which is the connected part of $\left\langle u_{\|}^{4}\right\rangle$. We can generalize this expression and introduce 3 variance and 3 curtosis terms separately for 00,01 , and 11 terms, and we can relate the kurtosis terms $\tau_{L L}$ 's to the parameters $\alpha_{L L}$ 's defined above as $2\left(\tau_{L L^{\prime}} / \sigma_{L L^{\prime}}\right)^{4}=1 / \alpha_{L L^{\prime}}$.

Let us summarize this discussion: the decoupling of small scale velocity dispersion like terms from the long range correlations motivates a resummation of the terms into the so called FoG kernels, which multiply the long range correlation terms contained in density-density $\left(P_{00}\right)$, density-momentum $\left(P_{01}\right)$ and momentum-momentum $\left(P_{11}\right)$ correlators. Only a portion of the terms can be motivated in such a way, while other terms are simply nonlinear couplings that do not reduce to linear order correlation on large scales. It is therefore difficult to provide a formal justification for this resummation, but it is worth analyzing to what extent this approach is useful. Here we use the distribution function expansion in equation (2.3) to formally define FoG kernel parameters: up to $(k \mu)^{5}$ this is equivalent to the exact expansion, but requires 3 different FoG kernels. An additional point that needs to be emphasized is that these quantities as we defined are a function of angle and scale, i.e. they are $\mu$ and $k$ dependent. Below we use numerical simulations to compare the original expansion in equation (2.3) to the FoG resummation version to see to what extent FoG approach is useful for the general treatment of RSD.

\subsection{Testing the Fingers-of-God model}

In this subsection we compare the FoG resummation of the higher order terms discussed above to numerical simulations. The upper panels of figure 6 show velocity dispersions determined from our simulations using equations (4.7) - (4.9). For clarity we plot the spherically-averaged velocity dispersions, i.e., those obtained from the monopole spectra. While the 3 velocity dispersion terms are similar to each other, they differ in the amplitude and scale dependence, suggesting that it is important to independently consider the FoG kernel for each spectrum in the nonlinear Kaiser formula. Note that these values are higher than those determined by previous studies (e.g., figure 7 of [47]). This is because our quantities are based on the mass-weighted velocities in contrast to the volume-weighted velocities discussed in [47]. We discuss the latter in section 4.2. 


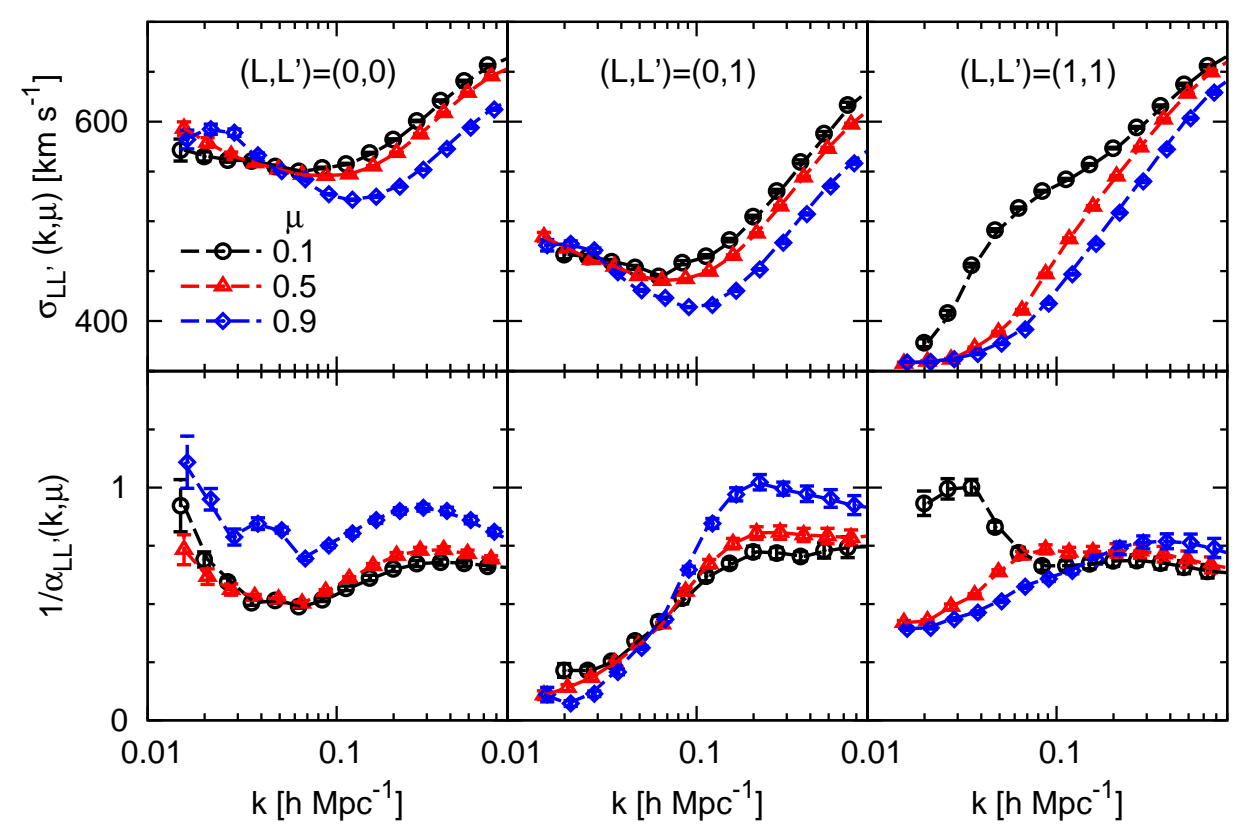

Figure 7. Angular dependence of FoG parameters at $z=0$. The width of $\mu$ bin is 0.2 centered around the values shown in the top left panel.

The lower panels in figure 6 show the inverses of the second parameters for our FoG model, $1 / \alpha_{L L^{\prime}}(k)$ defined by equation (4.12). As we have discussed above, the parameters are equivalent to $2\left(\tau_{L L^{\prime}} / \sigma_{L L^{\prime}}\right)^{4}$ when we adopt the kurtosis as the second parameters. Note that for a Lorentzian model and a Gaussian model we have $1 / \alpha_{L L^{\prime}}=1$ and $1 / \alpha_{L L^{\prime}}=0$, respectively. The impression from the figure is that $\alpha$ parameters have strong $k$ dependences and behave differently from each other at large scales. However, the FoG terms have negligibly small effects on the shape of the power spectrum at large scales. On the other hand, at lower redshifts the $\alpha$ values converge to nearly a constant at $k>0.1 \mathrm{~h} \mathrm{Mpc}^{-1}$ where the FoG effect starts to play an important role, with typical values between $1<\alpha_{L L^{\prime}}<2$, i.e. Lorentzian FoG kernel is a better approximation than Gaussian. The convergence of $\alpha_{L L}$, to a single value is worse as we go to higher redshifts and the difference between the values of $\alpha_{L L}$ 's remains large. In order to see the angular dependence of our FoG parameters, we show $\sigma_{L L^{\prime}}$ and $1 / \alpha_{L L^{\prime}}$ at $z=0$ as functions of $k$ and $\mu$ at the upper and lower panels in figure 7 , respectively.

Now we focus on the power spectrum $P^{s s}$ using the FoG model discussed above. In figures 2, 4 and 5 the resulting power spectra with the FoG kernels are shown as the red points. As we have seen in section 3.2, there were certain scales at which the power spectrum from our perturbative expansion approach breaks down and diverse, $k \simeq 0.2 h \mathrm{Mpc}^{-1}$ at $z=0$ and $k \simeq 0.4 h \mathrm{Mpc}^{-1}$ at $z=2$ for the monopole spectrum even though we sum up to the 6th order terms. Our FoG model dramatically improves the results. The accuracy of a few percent is achieved up to $k \simeq 0.4 h \mathrm{Mpc}^{-1}$ at $z=0$ and even up to to $k<1.0 h \mathrm{Mpc}^{-1}$ at $z=0.5$ and 1. On the other hand, the improvement at $z=2$ is not so much better than the lower redshfits because of a strong suppression by the FoG. Our FoG model works for the quadrupole spectrum as well as for the monopole spectrum: it predicts the quadrupole spectrum down to very small scales (see the upper panels of figure 4 and the right panels of figure 3). Even the accuracy of the hexadecapole spectrum of our FoG model is quite good, at the level of $10 \%$, and it reproduces the shape over all the scales probed (see upper panel of figure 5).

\subsection{Mass-weighted vs volume-weighted velocities}

It is worth comparing our approach based on the power spectra of mass-weighted velocity moments with those of volume-weighted velocity moments, which are commonly used to model nonlinear power spectra (e.g., $[25,32,47,62,63])$. The difference comes from the fact that the $L$-th moment of the 
mass-weighted moments $T_{\|}^{L}=(1+\delta) u_{\|}^{L}$ in equation (2.1) contains contributions not only from $L$-th order in perturbation theory, but also $(L+1)$-th order because of the term $\delta(\mathbf{x}) u_{\|}^{L}(\mathbf{x})$. By regrouping equation (2.1) with the same order term, we obtain

$$
\delta_{s}(k, \mu)=\delta(k, \mu)+\frac{i k_{\|}}{\mathcal{H}} u_{\|}(k, \mu)+\sum_{L=2}^{\infty}\left[\frac{1}{(L-1) !}\left(\frac{i k_{\|}}{\mathcal{H}}\right)^{L-1}\left[\delta(\mathbf{x}) u_{\|}^{L-1}(\mathbf{x})\right]_{\mathbf{k}}+\frac{1}{L !}\left(\frac{i k_{\|}}{\mathcal{H}}\right)^{L} u_{\|}^{L}(\mathbf{k})\right]
$$

where the first and the second terms of the right-hand side are respectively the zeroth and first order terms, while the bracketed terms correspond to the $L$-th order terms $(L \geq 2)$. By squaring equation (4.14), we obtain the same equation as equation (2.3), but the terms regrouped into the same order in SPT,

$$
P^{s s}(\mathbf{k})=P_{00}+2 \frac{i k \mu}{H} P_{0 u_{\|}^{1}}+\frac{(k \mu)^{2}}{H^{2}} P_{u_{\|}^{1} u_{\|}^{1}}+\left[2 \frac{i k \mu}{H} P_{0 w_{\|}^{1}}-\frac{(k \mu)^{2}}{H^{2}} P_{0 u_{\|}^{2}}\right]+\cdots
$$

where $w_{\|}^{L}=T_{\|}^{L}-u_{\|}^{L}=\left[\delta u_{\|}^{L}\right]_{\mathbf{k}}$, thus $P_{0 w_{\|}^{L}}=P_{0 L}-P_{0 u_{\|}^{L}}$ and similarly for higher orders.

As many previous studies have already discussed (e.g. [63]), measuring the power spectrum of volume-weighted velocity moments is not as straight-forward as measuring mass-weighted velocity moments used in our formalism. In order to measure the moments of volume-weighted velocities, we divide the interpolated moments of mass-weighted velocities by the interpolated density before the field is Fourier-transformed $T_{\|}^{L} /(1+\delta)=u_{\|}^{L}$. This can be noisy: some points on the grid may not have any particles, so a sufficiently coarse grid is needed. More importantly, the results depend on the grid size, i.e. on smoothing (see $[32,64]$ for a detailed discussion of how to measure the volumeweighted velocities from $N$-body simulations). Following the same process as described in section 3.1, we compute the redshift-space power spectrum based on the power spectra of volume-weighted velocity moments up to a given level of accuracy.

The top set of figure 8 shows the ratio of the summed power spectra of equation (4.15) to the corresponding reference spectra in redshift space. Because equation (4.15) is essentially the same as equation (2.3), here we want to see the convergence of these expressions. At the order of first 3 (Kaiser) terms, the expansion with volume-weighted velocities is somewhat closer to the reference spectrum than that with mass-weighted velocities, but both approximations are bad. The bottom set of figure 8 shows the same quantities as in the top set but those at $k=0.117 h \mathrm{Mpc}^{-1}$ at from 3 rd to 6 th orders. At higher orders the convergence is slightly faster with mass-weighted quantities for monopoles when they are compared at the same order: one can predict well the redshift-space power spectrum at $k=0.1 \mathrm{~h} \mathrm{Mpc}^{-1}$ by including the 4 th and 3rd order corrections at $z=0$ and $z>0$, respectively, for our power spectrum of mass-weighted velocity moments, while one needs to include the 4th order corrections for the power spectrum of volume-weighted velocity moments. For the quadrupole spectra, the situation is more complicated but the value of the spectra from the volume-weighted velocity moments expansion is deviated from the reference value at 3rd order at very large scales as seen at the bottom panels of the top set in figure 8. Both approaches break down once one enters the non-perturbative regime $\left(k>0.2 \mathrm{~h} \mathrm{Mpc}^{-1}\right.$ at $\left.z=0\right)$. We conclude that in terms of rate of convergence, there is no advantage in defining volume weighted quantities. If one works with galaxies and halos number density weighting becomes essential, since it is difficult to define volume weighted velocity moments in a sparsely sampled system [63]. In that situation our approach is the most meaningful way to define physical quantities that enter in RSD description.

\section{Expansion in powers of $\mu^{2}$}

As we have seen in previous two sections the series expansion of equation (2.3) is convergent on large scales, but not on small scales. For sufficiently high $k$ any finite order summation fails drastically. FoG resummation approach fares better, but even that fails for high $k$. One can sidestep these issues by considering an alternative expansion in terms of powers of $\mu^{2}$ : as discussed in section 2.1 and in 

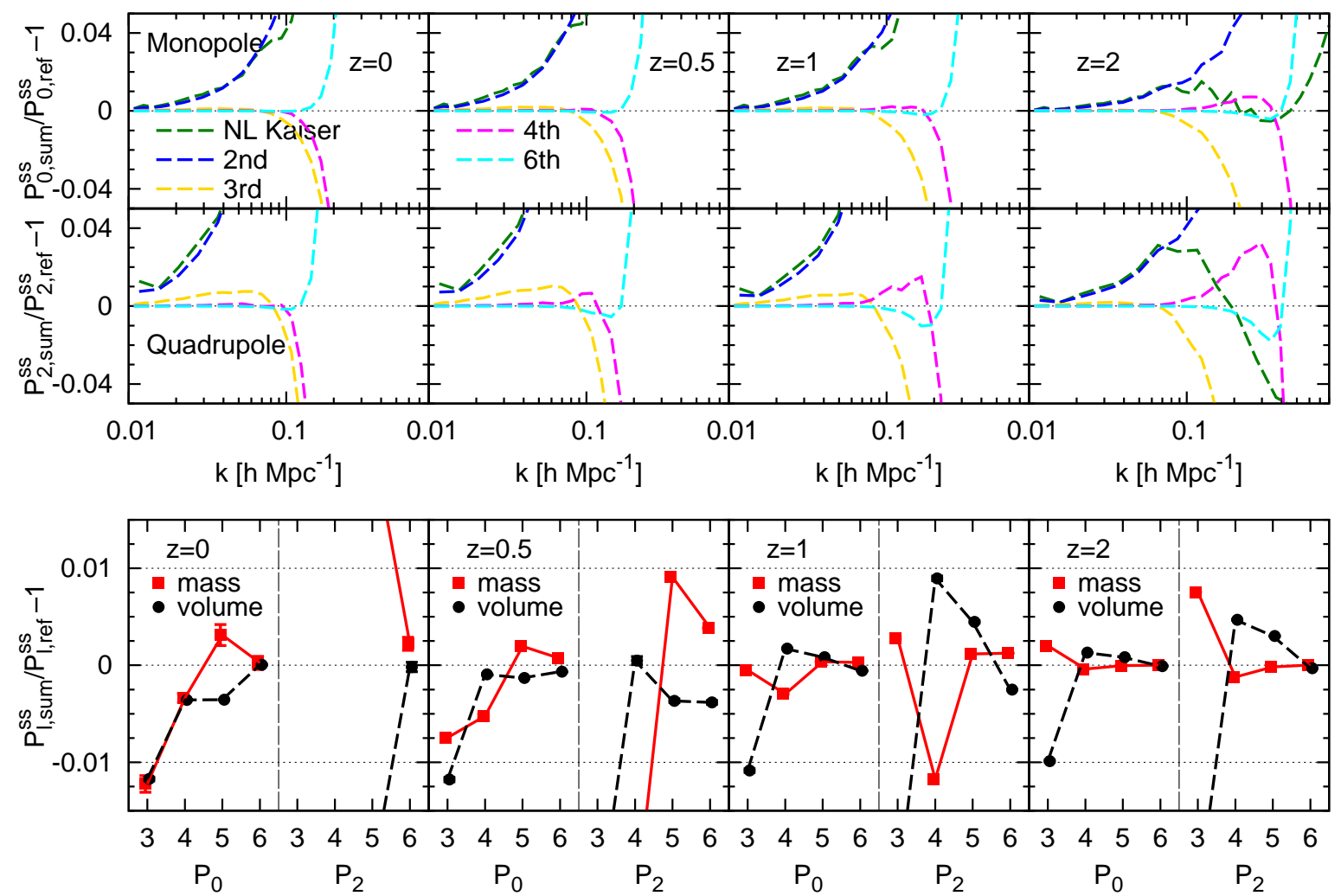

Figure 8. (Top set) Error between the summed power spectrum in terms of volume weighted velocity moment expansion and the reference spectrum. Lines are the same as the lower panels of figures 2 and 4 . (Bottom set) Error between the summed power spectrum and the reference spectrum at $k=0.117 h \mathrm{Mpc}^{-1}$. The left and right sides of each panel show the monopole and quadrupole spectra, respectively. The horizontal axis is a given order in terms of mass-weighted moment expansion (red points) and volume-weighted moment expansion (black points).

[49] for any finite power of $\mu^{2}$ there is a finite number of $P_{L L^{\prime}}$ terms contributing to it. For $\mu^{0}$ only $P_{00}$ contributes, for $\mu^{2} P_{01}, P_{11}$ and $P_{02}$, for $\mu^{4} P_{11}, P_{02}, P_{03}, P_{12}, P_{04}, P_{13}$ and $P_{22}$ etc.

Only these 3 lowest terms, $\mu^{0}, \mu^{2}$, and $\mu^{4}$, contain cosmological information at the linear order, so in principle these are the only relevant terms. However, if we expand the full $P^{s s}(k, \mu)$ into powers of $\mu^{2}$ and try to determine the coefficients from the data, the resulting coefficients will be correlated: only Legendre expansion assures uncorrelated values. As a result there will be mixing of higher powers of $\mu^{2}$ into lower powers if they are not accounted for in the fits, or there will be strong degeneracies and the fits will be unstable if all the coefficients are accounted for but we allow them to take any value. Typically one solves this by regularizing the expansion, i.e. by constraining them to a certain range of values. In this paper we will not focus on methods how to determine the coefficients of such expansion from the data, but we will show $\mu^{6}$ and $\mu^{8}$ expansion terms to develop some understanding of their scale dependence and amplitude.

\section{$5.1 \quad \mu^{2}$ terms}

In the top panels of figure 9 we show these individual term contributions to the lowest order powers of $\mu$ (we do not show $\mu^{0}$ term, which is just the usual real space power spectrum $P_{00}$ ). For $\mu^{2}$ we see that the $P_{01}$ dominates for low $k$, as expected since that is the only term that does not vanish in linear theory. This term follows linear theory prediction for low $k$, while for $k>0.1 h \mathrm{Mpc}^{-1}$ it exceeds it, just like it happens for the dark matter power spectrum $P_{00}$ itself. This is not surprising: as shown 


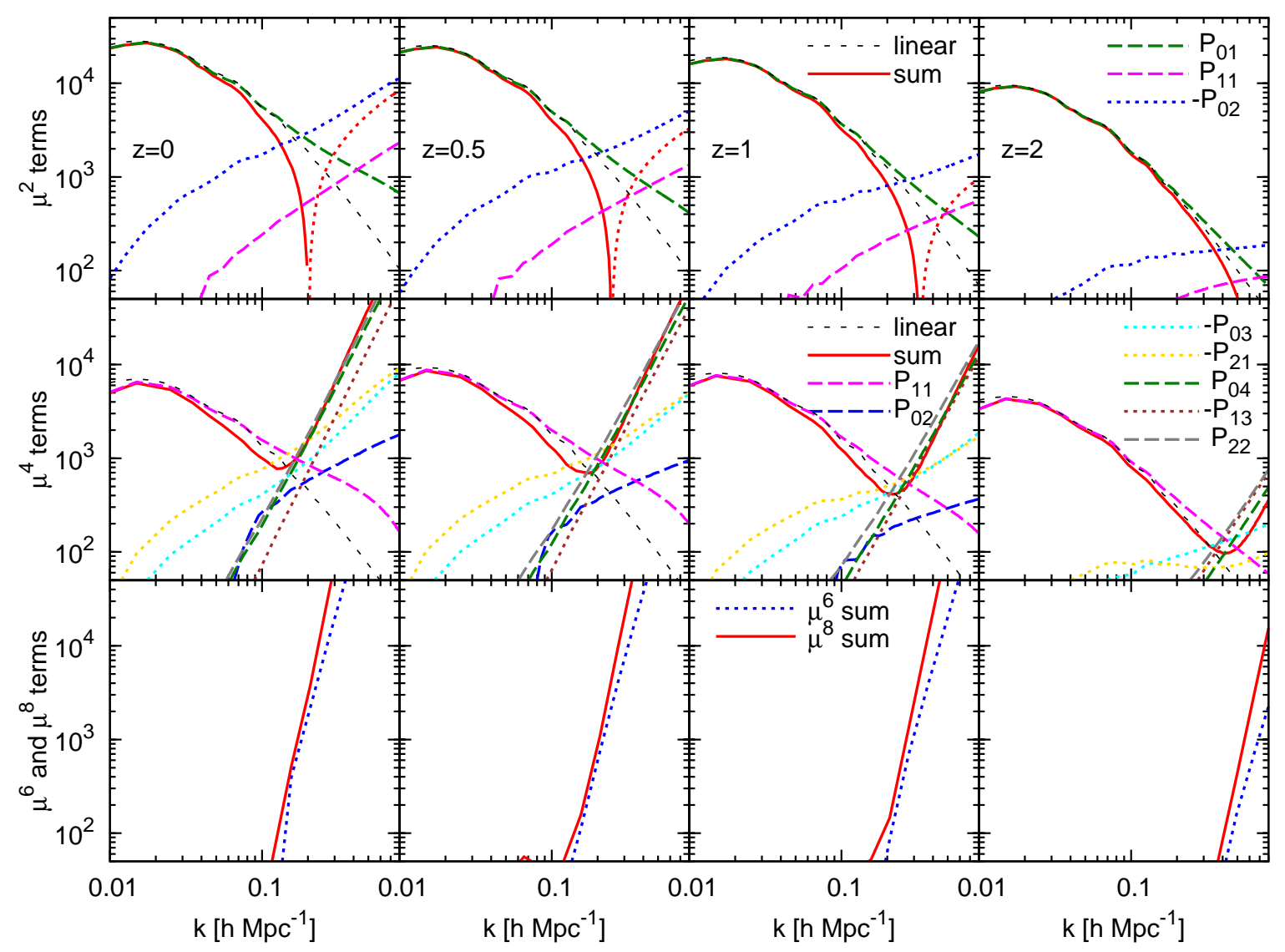

Figure 9. Contributions to $\mu^{2}$ (upper panels), $\mu^{4}$ (middle panels), and $\mu^{6}$ and $\mu^{8}$ terms (lower panels) in redshift-space power spectrum $P^{s s}(k, \mu)$. The positive contributions are shown as the solid and long-dashed lines while the negative contributions as the dotted lines (linear theory predictions are shown as the shortdashed black line).

in [49] we can write $P_{01}^{s s}\left(\mu^{2}\right)=\frac{d P_{00}(k)}{d \ln a}$, so this term is given by the time derivative of the dark matter power spectrum and has a similar scale dependence relative to the linear power spectrum.

The next term in terms of relevance is $P_{02}$. This term has contributions from the correlation between the energy density and the density $P_{00}^{020}$, as well as from the scalar part of the anisotropic stress correlated with the density $P_{02}^{020}$, both helicity 0 scalars. As discussed in section 4 , we expect the first term to be dominant and scale as $-k^{2} P_{00} \sigma^{2} / H^{2}$, hence to dominate over $P_{01}$ for $k \sigma / H>1$. This gives $\sigma=500 \mathrm{~km} / \mathrm{s}$ at $z=0$, decreasing to $200 \mathrm{~km} / \mathrm{s}$ for $z=2$. As expected we see this term is always negative.

The third term contributing to $\mu^{2}$ is the vector part (helicity 1 ) of auto-correlation of momentum density with itself $P_{11}^{111}$. This term is always positive and partially cancels $P_{02}$. As discussed in [49], it cancels all the bulk motion contributions to $\sigma^{2}$. While this term scales in a similar way as $P_{02}$, it is 3-10 times lower in amplitude, so it cancels only a small part of $P_{02}$.

We see that the total sum never exceeds the linear power spectrum and becomes negative for $k \sim 0.17 h \mathrm{Mpc}^{-1}$ at $z=0$ and $k \sim 0.5 h \mathrm{Mpc}^{-1}$ at $z=2$. For scales smaller than that the $\mu^{2}$ term is negative as a consequence of a strong FoG effect.

\section{$5.2 \quad \mu^{4}$ terms}

This term receives contributions from 7 different terms, $P_{11}, P_{02}, P_{03}, P_{12}, P_{04}, P_{13}$ and $P_{22}$. They are shown at the middle panels in figure 9 . On large scales the dominant term is $P_{11}$ which contains 
a linear order contribution. This term agrees with linear theory prediction for $k<0.1 h \mathrm{Mpc}^{-1}$ and is above that for $k>0.1 \mathrm{~h} \mathrm{Mpc}^{-1}$, just like in the case of $P_{00}$ and $P_{01}$.

The next order term in significance should be $P_{02}$. We see this term is relatively small and does not dominate anywhere. $P_{02}$ contribution to $\mu^{4}$ arises entirely from from the scalar part of the anisotropic stress correlated with the density $P_{02}^{020}$, which contains $\mu^{4}$ term contribution to $P^{s s}$, while $P_{00}^{020}$ does not. Physically one expects the small scale velocity dispersion to be relatively isotropic, hence anisotropic stress density should be small compared to the energy density.

Next two terms in terms of $L+L^{\prime}$ are $P_{03}$ and $P_{12}$. These two terms provide the dominant correction to $P_{11}$ on intermediate and large scales $k<0.1 h \mathrm{Mpc}^{-1}$. On very small scales terms $P_{04}$, $P_{22}$ and $P_{13}$ dominate. As discussed in FoG section we expect the first two terms to scale as $3 k^{4} \sigma^{4} P_{00}$ and be equal in amplitude, while $P_{13}$ should be negative and cancel out the bulk flow part of the other two terms. We see that this expectation is borne out in simulations: in total these terms add power on small scales. The transition happens at a similar scale as for $\mu^{2}$ term, $k \sim 0.17 \mathrm{~h} \mathrm{Mpc}^{-1}$ at $z=0$ and $k \sim 0.5 h \mathrm{Mpc}^{-1}$ at $z=2$.

\subsection{Higher order terms}

At order higher than $\mu^{4}$ we do not have any linear order contributions, so we expect these terms to be small on large scales. There are many terms that contribute. At the bottom panels in figure 9 we show the total contributions to $\mu^{6}$ and $\mu^{8}$ terms. We can see that these terms are indeed negligibly small at $k<0.1 h \mathrm{Mpc}^{-1}$ at $z=0$ and $k<0.3 h \mathrm{Mpc}^{-1}$ at $z=2$. At smaller scales these contributions increase with the scale dependences of $k^{6}$ and $k^{8}$, respectively.

\section{Conclusions}

In this paper we used numerical simulations to investigate the distribution function expansion approach to the redshift space distortion power spectrum [49]. The power spectrum in redshift space can be written as a sum over correlators between mass-weighted velocity moments. We analyzed a large set of cosmological $N$-body simulations to test how accurately this formalism predicts the true power spectrum in redshift space and how many terms are needed to achieve a given precision. We compared to the RSD power spectrum as a function of wavevector $k$ and cosine of the angle between the Fourier mode and line of sight $\mu$, as well as to the lowest Legendre moments, monopole, quadrupole, and hexadecapole. These comparisons revealed that the expansion is accurate within a few percent up to $k \simeq 0.2 h \mathrm{Mpc}^{-1}$ at $z=0$ and $k \simeq 0.3 h \mathrm{Mpc}^{-1}$ at $z=2$, if the corrections up to the 6th order are taken into account. All expansions break down at higher $k$, where the expansion parameter becomes larger than unity.

The expansion can be resummed into power suppression factors known as the Finger-of-God (FoG) kernels. Our expansion formula suggests that one needs three independent FoG kernels, each of which is multiplying the lowest 3 terms in the expansion, which are the 3 terms that contain linear order contributions (Kaiser formula). We have found that the 3 velocity dispersions differ in their values. We also found that the shape of the FoG kernels differ and are a function of scale, redshift and angle. There is no single FoG kernel that would fit the simulation data. This FoG model has validity comparable or better than the highest (6th) order summations we have tried. Our FoG model predicts the monopole power spectrum with a few percent accuracy up to $k \simeq 0.4 h \mathrm{Mpc}^{-1}$ at $z=0$ and is accurate for $k<1 h \mathrm{Mpc}^{-1}$ at $z=0.5$ and 1 .

The difficulty of obtaining convergence or a perfect FoG model on small scales in Legendre moments expansion do not exist in the alternative expansion in powers of $\mu^{2 j}$. This expansion has the advantage of containing a finite number of terms for a given value of $j$, and hence no infinite sums are needed, there is always convergence for any finite $j$. In contrast every Legendre moment receives infinite number of terms. We have used the angular decomposition of [49] to derive the individual terms for the lowest values of $j$. We compared their amplitudes as a function of scale, showing that for $\mu^{2}$ and $\mu^{4}$ the nonlinear FoG terms dominate the Kaiser terms for $k>0.2 h \mathrm{Mpc}^{-1}(z=0)$. Similarly, higher orders $\left(\mu^{6}\right.$ etc), which do not receive any contributions at the linear order, are nearly zero for $k \ll 0.2 h \mathrm{Mpc}^{-1}$, but grow very rapidly on smaller scales. Because the higher order terms 
of $P_{L L^{\prime}}$ also have higher order powers of $\mu^{2}$, one can in principle separate them from lower order terms, put them all into terms that we do not care about, and we can marginalize over. Note that the expansion in powers of $\mu^{2}$ has the disadvantage that the coefficients extracted from observations will be correlated, unlike Legendre moments, which are orthogonal, at least on scales small compared to the size of the survey. In practice this means that some priors will have to be assumed for the terms with higher powers of $\mu^{2}$, at least at high $k$, otherwise they will contaminate the determination of $\mu^{0}$, $\mu^{2}$ and $\mu^{4}$ terms. In this sense we cannot escape the high order FoG complications. However, it is not clear there is any useful information remaining at $k>0.2 h \mathrm{Mpc}^{-1}(z=0)$ due to the nonlinear evolution anyways. This will be explored further with perturbation theory in paper III of this series [65].

We also compared our approach based on the power spectrum of mass-weighted velocity moments to the one based on the power spectrum of volume-weighted velocity moments. We found that the power spectrum based on the mass-weighted velocity moments converges slightly faster, although the difference is not so significant. The fact that there is no natural way to expand RSD into volume weighted quantities, and that using volume weighted quantities does not improve the convergence, implies that there is no good reason to work with volume weighted quantities in RSD studies.

This advantage of mass weighting quantities becomes crucial when the same formalism is applied to galaxies and halos, where volume weighted moments cannot be easily defined. It is difficult or impossible to measure the velocity power spectrum from the sparse density field and the results depend on the smoothing (see e.g. [63] for an attempt and failure to measure it). In contrast to this, the analysis presented in this paper can be naturally extended to the clustering analysis of halos and galaxies, since density weighted moments are not affected by sparseness of the sample. Recently it was shown by [24] (but see also [66]) that the RSD parameters reconstracted from redshift-space distortions of dark matter halos have strong halo-mass and scale dependence even on large scales $\left(k<0.1 h \mathrm{Mpc}^{-1}\right)$. This can be a serious problem when one wants to constrain dark energy or modified gravity theories. This complicated scale dependence is likely to be a consequence of the number density weighting of velocity moments, which differs from the mass weighting of dark matter if galaxies or halos have a different spatial distribution. The scale dependence will arise even for a linear bias model, except on very large scales, where galaxy overdensity $\delta_{g} \ll 1[49,67]$. The formalism presented in this paper can be used to investigate this scale dependence and we plan to present this analysis in paper IV of this series.

\section{Acknowledgments}

We would like to thank Nico Hamaus, Zvonimir Vlah and Tobias Baldauf for help and useful discussions. This research was supported by the DOE, and the Swiss National Foundation under contract 200021-116696/1 and Republic of Korea WCU grant R32-10130.

\section{A Derivatives of FoG kernels from higher-order $P_{L L^{\prime}}$ 's}

In this appendix, we present derivatives of the FoG kernels: $G_{00}, G_{01}$ and $G_{11}$ for the auto power spectrum of the redshift-space density field,

$$
\begin{aligned}
\left.G_{00}^{(1)}\left(x_{00}\right)\right|_{x_{00}=0} & =-\frac{1}{\sigma_{00}^{2}} \frac{P_{02}}{P_{00}} \\
\left.G_{01}^{(1)}\left(x_{01}\right)\right|_{x_{01}=0} & =-\frac{1}{\sigma_{01}^{2} P_{01}}\left[\frac{1}{6} P_{03}+\frac{1}{2} P_{21}\right] \\
\left.G_{11}^{(1)}\left(x_{11}\right)\right|_{x_{11}=0} & =-\frac{1}{3 \sigma_{11}^{2}} \frac{P_{13}}{P_{11}} \\
\left.G_{00}^{(2)}\left(x_{00}\right)\right|_{x_{00}=0} & =\frac{1}{\sigma_{00}^{4} P_{00}}\left[\frac{1}{6} P_{04}+\frac{1}{2} P_{22}\right] \\
\left.G_{01}^{(2)}\left(x_{01}\right)\right|_{x_{01}=0} & =\frac{1}{\sigma_{01}^{4} P_{01}}\left[\frac{1}{60} P_{05}+\frac{1}{12} P_{41}+\frac{1}{6} P_{23}\right]
\end{aligned}
$$




$$
\begin{aligned}
\left.G_{11}^{(2)}\left(x_{11}\right)\right|_{x_{11}=0} & =\frac{1}{\sigma_{11}^{4} P_{11}}\left[\frac{1}{30} P_{15}+\frac{1}{16} P_{33}\right], \\
\left.G_{00}^{(3)}\left(x_{00}\right)\right|_{x_{00}=0} & =-\frac{1}{\sigma_{00}^{6} P_{00}}\left[\frac{1}{60} P_{06}+\frac{1}{4} P_{24}\right], \\
\left.G_{01}^{(3)}\left(x_{01}\right)\right|_{x_{01}=0} & =-\frac{1}{\sigma_{01}^{6} P_{01}}\left[\frac{1}{840} P_{07}+\frac{1}{120} P_{61}+\frac{1}{40} P_{25}+\frac{1}{24} P_{43}\right], \\
\left.G_{11}^{(3)}\left(x_{11}\right)\right|_{x_{11}=0} & =-\frac{1}{\sigma_{11}^{6} P_{11}}\left[\frac{1}{420} P_{17}+\frac{1}{60} P_{35}\right], \\
\left.G_{00}^{(4)}\left(x_{00}\right)\right|_{x_{00}=0} & =\frac{1}{\sigma_{00}^{8} P_{00}}\left[\frac{1}{840} P_{08}+\frac{1}{30} P_{26}+\frac{1}{24} P_{44}\right], \\
\left.G_{01}^{(4)}\left(x_{01}\right)\right|_{x_{01}=0} & =\frac{1}{\sigma_{11}^{8} P_{01}}\left[\frac{1}{15120} P_{09}+\frac{1}{1680} P_{81}+\frac{1}{420} P_{27}+\frac{1}{180} P_{63}+\frac{1}{120} P_{45}\right], \\
\left.G_{11}^{(4)}\left(x_{11}\right)\right|_{x_{11}=0} & =\frac{1}{\sigma_{11}^{8} P_{11}}\left[\frac{1}{7560} P_{19}+\frac{1}{630} P_{37}+\frac{1}{600} P_{55}\right] .
\end{aligned}
$$

Note that these equations are general and not dependent of the ansatz of the kernel (equation (4.10)). As discussed in section 4, the first derivatives of $G_{L L^{\prime}}$ give the definitions of the velocity dispersions $\sigma_{L L^{\prime}}$. The power low index parameters of our FoG model, $\alpha_{L L^{\prime}}$, are obtained by substituting equations (A.4) - (A.6) into equation (4.12).

\section{References}

[1] P. J. E. Peebles, The large-scale structure of the universe (1980).

[2] D. J. Eisenstein, I. Zehavi, D. W. Hogg, R. Scoccimarro, M. R. Blanton, R. C. Nichol, R. Scranton, H. Seo, M. Tegmark, Z. Zheng, et al., Astrophys. J. 633, 560 (Nov. 2005), arXiv:astro-ph/0501171.

[3] S. Cole, W. J. Percival, J. A. Peacock, P. Norberg, C. M. Baugh, C. S. Frenk, I. Baldry, J. Bland-Hawthorn, T. Bridges, R. Cannon, et al., Mon. Not. Roy. Astron. Soc. 362, 505 (Sep. 2005), arXiv:astro-ph/0501174.

[4] J. C. Jackson, Mon. Not. Roy. Astron. Soc. 156, 1P (1972).

[5] N. Kaiser, Mon. Not. Roy. Astron. Soc. 227, 1 (Jul. 1987).

[6] A. J. S. Hamilton, in D. Hamilton, ed., The Evolving Universe (1998), vol. 231 of Astrophysics and Space Science Library, pp. 185-+, arXiv:astro-ph/9708102.

[7] E. V. Linder, Phys. Rev. D 72(4), 043529 (Aug. 2005), arXiv:astro-ph/0507263.

[8] B. Jain and P. Zhang, Phys. Rev. D 78(6), 063503 (Sep. 2008), 0709.2375.

[9] Y. Song and K. Koyama, JCAP 1, 48 (Jan. 2009), 0802.3897.

[10] C. Alcock and B. Paczynski, Nature 281, 358 (Oct. 1979).

[11] T. Matsubara and Y. Suto, Astrophys. J. Let. 470, L1+ (Oct. 1996), arXiv:astro-ph/9604142.

[12] W. E. Ballinger, J. A. Peacock, and A. F. Heavens, Mon. Not. Roy. Astron. Soc. 282, 877 (Oct. 1996), arXiv:astro-ph/9605017.

[13] J. A. Peacock, S. Cole, P. Norberg, C. M. Baugh, J. Bland-Hawthorn, T. Bridges, R. D. Cannon, M. Colless, C. Collins, W. Couch, et al., Nature 410, 169 (Mar. 2001), arXiv:astro-ph/0103143.

[14] I. Zehavi, M. R. Blanton, J. A. Frieman, D. H. Weinberg, H. J. Mo, M. A. Strauss, S. F. Anderson, J. Annis, N. A. Bahcall, M. Bernardi, et al., Astrophys. J. 571, 172 (May 2002), arXiv:astro-ph/0106476.

[15] E. Hawkins, S. Maddox, S. Cole, O. Lahav, D. S. Madgwick, P. Norberg, J. A. Peacock, I. K. Baldry, C. M. Baugh, J. Bland-Hawthorn, et al., Mon. Not. Roy. Astron. Soc. 346, 78 (Nov. 2003), arXiv:astro-ph/0212375. 
[16] M. Tegmark, M. A. Strauss, M. R. Blanton, K. Abazajian, S. Dodelson, H. Sandvik, X. Wang, D. H. Weinberg, I. Zehavi, N. A. Bahcall, et al., Phys. Rev. D 69(10), 103501 (May 2004), arXiv:astro-ph/0310723.

[17] M. Tegmark, D. J. Eisenstein, M. A. Strauss, D. H. Weinberg, M. R. Blanton, J. A. Frieman, M. Fukugita, J. E. Gunn, A. J. S. Hamilton, G. R. Knapp, et al., Phys. Rev. D 74(12), 123507 (Dec. 2006), arXiv:astro-ph/0608632.

[18] N. P. Ross, J. da Ângela, T. Shanks, D. A. Wake, R. D. Cannon, A. C. Edge, R. C. Nichol, P. J. Outram, M. Colless, W. J. Couch, et al., Mon. Not. Roy. Astron. Soc. 381, 573 (Oct. 2007), arXiv:astro-ph/0612400.

[19] L. Guzzo, M. Pierleoni, B. Meneux, E. Branchini, O. Le Fèvre, C. Marinoni, B. Garilli, J. Blaizot, G. De Lucia, A. Pollo, et al., Nature 451, 541 (Jan. 2008), 0802.1944.

[20] T. Okumura, T. Matsubara, D. J. Eisenstein, I. Kayo, C. Hikage, A. S. Szalay, and D. P. Schneider, Astrophys. J. 676, 889 (Apr. 2008), 0711.3640.

[21] A. Cabré and E. Gaztañaga, Mon. Not. Roy. Astron. Soc. 393, 1183 (Mar. 2009), 0807.2460.

[22] C. Blake, S. Brough, M. Colless, C. Contreras, W. Couch, S. Croom, T. Davis, M. J. Drinkwater, K. Forster, D. Gilbank, et al., Mon. Not. Roy. Astron. Soc. pp. 834-+ (Jun. 2011), 1104.2948.

[23] J. L. Tinker, D. H. Weinberg, and Z. Zheng, Mon. Not. Roy. Astron. Soc. 368, 85 (May 2006), arXiv:astro-ph/0501029.

[24] T. Okumura and Y. P. Jing, Astrophys. J. 726, 5 (Jan. 2011), 1004.3548.

[25] E. Jennings, C. M. Baugh, and S. Pascoli, Mon. Not. Roy. Astron. Soc. 410, 2081 (Jan. 2011), 1003.4282

[26] J. Kwan, G. F. Lewis, and E. V. Linder, ArXiv e-prints (May 2011), 1105.1194.

[27] G. J. Hill, K. Gebhardt, E. Komatsu, and P. J. MacQueen, in R. E. Allen, D. V. Nanopoulos, \& C. N. Pope, ed., The New Cosmology: Conference on Strings and Cosmology (Dec. 2004), vol. 743 of American Institute of Physics Conference Series, pp. 224-233.

[28] K. Glazebrook, C. Blake, W. Couch, D. Forbes, M. Drinkwater, R. Jurek, K. Pimbblet, B. Madore, C. Martin, T. Small, et al., ArXiv Astrophysics e-prints (Jan. 2007), arXiv:astro-ph/0701876.

[29] D. Schlegel, M. White, and D. Eisenstein, in astro2010: The Astronomy and Astrophysics Decadal Survey (2009), vol. 2010 of ArXiv Astrophysics e-prints, pp. 314-+, 0902.4680.

[30] M. Sumiyoshi, T. Totani, S. Oshige, K. Glazebrook, M. Akiyama, T. Morokuma, K. Motohara, K. Shimasaku, M. Hayashi, M. Yoshida, et al., ArXiv e-prints (Feb. 2009), 0902.2064.

[31] D. J. Schlegel, C. Bebek, H. Heetderks, S. Ho, M. Lampton, M. Levi, N. Mostek, N. Padmanabhan, S. Perlmutter, N. Roe, et al., ArXiv e-prints (Apr. 2009), 0904.0468.

[32] R. Scoccimarro, Phys. Rev. D 70(8), 083007 (Oct. 2004), arXiv:astro-ph/0407214.

[33] F. Bernardeau, S. Colombi, E. Gaztañaga, and R. Scoccimarro, Phys. Rept. 367, 1 (Sep. 2002), arXiv:astro-ph/0112551.

[34] M. Crocce and R. Scoccimarro, Phys. Rev. D 73(6), 063519 (Mar. 2006), arXiv:astro-ph/0509418.

[35] M. Crocce and R. Scoccimarro, Phys. Rev. D 73(6), 063520 (Mar. 2006), arXiv:astro-ph/0509419.

[36] S. Matarrese and M. Pietroni, JCAP 6, 26 (Jun. 2007), arXiv:astro-ph/0703563.

[37] P. McDonald, Phys. Rev. D 75(4), 043514 (Feb. 2007), arXiv:astro-ph/0606028.

[38] P. Valageas, Astron. Astrophys. 465, 725 (Apr. 2007), arXiv:astro-ph/0611849.

[39] A. Taruya and T. Hiramatsu, Astrophys. J. 674, 617 (Feb. 2008), 0708.1367.

[40] T. Nishimichi, A. Shirata, A. Taruya, K. Yahata, S. Saito, Y. Suto, R. Takahashi, N. Yoshida, T. Matsubara, N. Sugiyama, et al., PASJ 61, 321 (Feb. 2009), 0810.0813.

[41] J. Carlson, M. White, and N. Padmanabhan, Phys. Rev. D 80(4), 043531 (Aug. 2009), 0905.0479.

[42] A. F. Heavens, S. Matarrese, and L. Verde, Mon. Not. Roy. Astron. Soc. 301, 797 (Dec. 1998), 
arXiv:astro-ph/9808016.

[43] R. Scoccimarro, H. M. P. Couchman, and J. A. Frieman, Astrophys. J. 517, 531 (Jun. 1999), arXiv:astro-ph/9808305.

[44] S. Bharadwaj, Mon. Not. Roy. Astron. Soc. 327, 577 (Oct. 2001), arXiv:astro-ph/0105320.

[45] B. Pandey and S. Bharadwaj, Mon. Not. Roy. Astron. Soc. 358, 939 (Apr. 2005), arXiv:astro-ph/0403670.

[46] T. Matsubara, Phys. Rev. D 77(6), 063530 (Mar. 2008), 0711.2521.

[47] A. Taruya, T. Nishimichi, and S. Saito, Phys. Rev. D 82(6), 063522 (Sep. 2010), 1006.0699.

[48] P. Valageas, Astron. Astrophys. 526, A67+ (Feb. 2011), 1009.0106.

[49] U. Seljak and P. McDonald, JCAP 11, 39 (Nov. 2011), 1109.1888.

[50] P. McDonald, JCAP 4, 32 (Apr. 2011), 0910.1002.

[51] V. Desjacques, U. Seljak, and I. T. Iliev, Mon. Not. Roy. Astron. Soc. 396, 85 (Jun. 2009), 0811.2748.

[52] U. Seljak and M. Zaldarriaga, Astrophys. J. 469, 437 (Oct. 1996), arXiv:astro-ph/9603033.

[53] E. Komatsu, J. Dunkley, M. R. Nolta, C. L. Bennett, B. Gold, G. Hinshaw, N. Jarosik, D. Larson, M. Limon, L. Page, et al., Astrophys. J. Sup. 180, 330 (Feb. 2009), 0803.0547.

[54] P. McDonald and U. Seljak, JCAP 10, 7 (Oct. 2009), 0810.0323.

[55] K. Yamamoto, B. A. Bassett, and H. Nishioka, Physical Review Letters 94(5), 051301 (Feb. 2005), arXiv:astro-ph/0409207.

[56] D. Tocchini-Valentini, M. Barnard, C. L. Bennett, and A. S. Szalay, ArXiv e-prints (Jan. 2011), 1101.2608.

[57] A. Taruya, S. Saito, and T. Nishimichi, Phys. Rev. D 83(10), 103527 (May 2011), 1101.4723.

[58] S. Cole, K. B. Fisher, and D. H. Weinberg, Mon. Not. Roy. Astron. Soc. 267, 785 (Apr. 1994), arXiv:astro-ph/9308003.

[59] D. J. Eisenstein and W. Hu, Astrophys. J. 496, 605 (Mar. 1998), arXiv:astro-ph/9709112.

[60] J. A. Peacock and S. J. Dodds, Mon. Not. Roy. Astron. Soc. 267, 1020 (Apr. 1994), arXiv:astro-ph/9311057.

[61] C. Park, M. S. Vogeley, M. J. Geller, and J. P. Huchra, Astrophys. J. 431, 569 (Aug. 1994).

[62] W. J. Percival and M. White, Mon. Not. Roy. Astron. Soc. 393, 297 (Feb. 2009), 0808.0003.

[63] J. Tang, I. Kayo, and M. Takada, Mon. Not. Roy. Astron. Soc. 416, 2291 (Sep. 2011), 1103.3614.

[64] S. Pueblas and R. Scoccimarro, Phys. Rev. D 80(4), 043504 (Aug. 2009), 0809.4606.

[65] Z. Vlah, U. Seljak, P. McDonald, T. Okumura, and T. Baldauf, in preparation .

[66] B. A. Reid and M. White, Mon. Not. Roy. Astron. Soc. 417, 1913 (Nov. 2011), 1105.4165.

[67] V. Desjacques and R. K. Sheth, Phys. Rev. D 81(2), 023526 (Jan. 2010), 0909.4544. 